\title{
The Timing for Neuronal Maturation in the Adult Hippocampus Is Modulated by Local Network Activity
}

\author{
Verónica C. Piatti, ${ }^{\star}$ M. Georgina Davies-Sala, ${ }^{\star}$ M. Soledad Espósito, ${ }^{\star}$ Lucas A. Mongiat, Mariela F. Trinchero, \\ and Alejandro F. Schinder \\ Laboratory of Neuronal Plasticity, Leloir Institute, Consejo Nacional de Investigaciones Científicas y Técnicas, 1405 Buenos Aires, Argentina
}

\begin{abstract}
The adult hippocampus continuously generates new cohorts of immature neurons with increased excitability and plasticity. The window for the expression of those unique properties in each cohort is determined by the time required to acquire a mature neuronal phenotype. Here, we show that local network activity regulates the rate of maturation of adult-born neurons along the septotemporal axis of the hippocampus. Confocal microscopy and patch-clamp recordings were combined to assess marker expression, morphological development, and functional properties in retrovirally labeled neurons over time. The septal dentate gyrus displayed higher levels of basal network activity and faster rates of newborn neuron maturation than the temporal region. Voluntary exercise enhanced network activity only in the temporal region and, in turn, accelerated neuronal development. Finally, neurons developing within a highly active environment exhibited a delayed maturation when their intrinsic electrical activity was reduced by the cell-autonomous overexpression of Kir2.1, an inward-rectifying potassium channel. Our findings reveal a novel type of activity-dependent plasticity acting on the timing of neuronal maturation and functional integration of newly generated neurons along the longitudinal axis of the adult hippocampus.
\end{abstract}

\section{Introduction}

The dentate gyrus of the adult hippocampus generates new dentate granule cells (DGCs) capable of information processing (Zhao et al., 2008). Adult neurogenesis is a complex process whereby neural progenitor cells adopt a neuronal fate, migrate, and mature over several weeks. During that period, young DGCs extend their axon and dendrites, establishing an appropriate input/output connectivity within the preexisting network (Ambrogini et al., 2004; Espósito et al., 2005; Overstreet-Wadiche et al., 2005, 2006a; Ge et al., 2006; Laplagne et al., 2006; Piatti et al., 2006; Toni et al., 2007, 2008). Interestingly, immature DGCs exhibit reduced GABAergic inhibition, high excitability, and a lower threshold for the induction of long-term potentiation (Wang et al., 2000; Snyder et al., 2001; Schmidt-Hieber et al., 2004; Espósito et al., 2005; Overstreet-Wadiche et al., 2005; Ge et al., 2006, 2007; Markwardt et al., 2009; Mongiat et al., 2009). Such

Received March 18, 2011; accepted April 5, 2011.

Author contributions: V.C.P., M.G.D.-S., M.S.E., and A.F.S. designed research; V.C.P., M.G.D.-S., M.S.E., L.A.M., and M.F.T. performed research; V.C.P., M.G.D.-S., M.S.E., L.A.M., M.F.T., and A.F.S. analyzed data; V.C.P. and A.F.S. wrote the paper.

This work was supported by the Guggenheim Foundation Fellowship and by Agencia Nacional para la Promoción de Ciencia y Tecnología Grant PICT2005, the American Health Assistance Foundation (Alzheimer's Disease Research Grant A2007-078), the Delfina Baratelli Foundation, and the Howard Hughes Medical Institute (International Scholars Program Grant 55005963) (A.F.S.). A.F.S. and L.A.M. are investigators of the Argentine National Research Council [Consejo Nacional de Investigaciones Científicas y Técnicas (CONICET)]. V.C.P., M.G.D.-S., and M.S.E. were supported by doctoral fellowships from CONICET. We thank Mariela Veggetti for technical assistance, and Guillermo Lanuza, Antonia Marín Burgin, and Laura Ewell for critical suggestions and for comments on this manuscript

The authors declare no competing financial interests.

*V.C.P., M.G.D.S., and M.S.E. contributed equally to this work.

Correspondence should be addressed to Alejandro F. Schinder, Laboratory of Neuronal Plasticity, Leloir Institute, Consejo Nacional de Investigaciones Científicas y Técnicas, Avenida Patricias Argentinas 435, 1405 Buenos Aires, Argentina. E-mail: aschinder@leloir.org.ar.

DOI:10.1523/JNEUROSCI.1380-11.2011

Copyright $\odot 2011$ the authors $\quad 0270-6474 / 11 / 317715-14 \$ 15.00 / 0$ high excitability allows them to spike despite their incipient glutamatergic inputs. Therefore, immature DGCs might participate in information processing before reaching a fully mature stage. After reaching maturity, their excitability decreases and they acquire a degree of functional connectivity and plasticity that is similar to that of all preexisting DGCs (Laplagne et al., 2006, 2007). Thus, new cohorts of immature DGCs with unique functional properties are continuously generated. The duration and the impact of those distinctive functional properties in the dentate gyrus network will depend on the speed of maturation of new DGCs.

The most well characterized regulatory steps of adult hippocampal neurogenesis are the rate of progenitor cell proliferation and the survival rate of new DGCs. For instance, exercise, learning, and enriched environment can increase neurogenesis, whereas aging, stress, and depression decrease it (Gould et al., 1999; van Praag et al., 1999; Parent and Lowenstein, 2002; Abrous et al., 2005; Zhao et al., 2008). These physiological stimuli primarily affect proliferation or survival. The notion that neuronal maturation can be altered was first highlighted by epileptic seizures, in which new DGCs showed mature features during early development (Overstreet-Wadiche et al., 2006b). Chronic antidepressant treatments were also shown to stimulate neuronal maturation (Wang et al., 2008). In addition, experimental manipulations that altered GABA signaling in new DGCs induced remarkable defects in their development (Ge et al., 2006). Those previous works involving a pathological condition or a specific alteration in GABA signaling showed that neuronal development is sensitive to altered activity. However, whether the timing of maturation of newborn DGCs can be regulated by changes that occur within a physiological range of neuronal activity remains unknown. 
The rodent hippocampus displays a longitudinal axis bending from the septal nuclei rostrodorsally toward the temporal lobe in the caudoventral area (see Fig. 1A) (Amaral and Witter, 1989). Despite the lack of anatomically defined regions, the patterns of anatomical connectivity and gene expression support a compartmentalization of the hippocampus along its longitudinal (septotemporal) axis (Verwer et al., 1997; Dolorfo and Amaral, 1998; Petrovich et al., 2001; Thompson et al., 2008; Fanselow and Dong, 2010). Consistently, distinct behaviors involve different areas of the hippocampus (Bast and Feldon, 2003; Bannerman et al., 2004). The septal to intermediate hippocampus receives connections from the dorsolateral entorhinal cortex and it is primarily associated to spatial learning (Moser et al., 1995; Fyhn et al., 2004). The temporal to intermediate hippocampus, via connections to medial prefrontal cortex and subcortical sites such as the amygdala and hypothalamus, is linked to anxiety and emotional state (Kjelstrup et al., 2002). Finally, the intermediate region exhibits overlapping characteristics with its neighbors and it has been involved in functional translation of rapid place learning to behavioral performance (Bast et al., 2009). In this context, the adult dentate gyrus itself may present different neurogenic environments for neuronal maturation. Interestingly, recent studies have revealed that the granule cell layer (GCL) of the septal pole of the dentate gyrus displays higher levels of basal neuronal activity than the remaining GCL. Moreover, the density of newborn neurons is also higher in the septal dentate gyrus (Snyder et al., 2009a,b; Jinno, 2011). Here, we demonstrate that those regional differences in basal activity can act as endogenous regulators of the rate of neuronal maturation.

\section{Materials and Methods}

Production of viral vectors. We used a retroviral vector based on the Moloney murine leukemia virus (Laplagne et al., 2006). Retroviral particles were assembled using three separate plasmids containing the envelope (CMV-vsvg), viral proteins (CMV-gag/pol), and transgenes. Transgenes included a fluorescent reporter such as GFP or RFP, mouse Kir2.1 (a gift from G. Lanuza, Instituto de Biología y Medicina Experimental, Buenos Aires, Argentina), or the nonconductive mutant $\operatorname{Kir}_{\mathrm{NC}}$ under CAG or PGK promoters. $\mathrm{Kir}_{\mathrm{NC}}$ was obtained by replacing residues $\mathrm{G}_{144} \mathrm{Y}_{145} \mathrm{G}_{146}$ to AAA in Kir2.1 using site-directed mutagenesis (Burrone et al., 2002). Kir or $\operatorname{Kir}_{\mathrm{NC}}$ sequences were followed by an internal ribosome entry site (IRES) and a fluorescent reporter (CAG-Kir-IRES-GFP and CAG$\mathrm{Kir}_{\mathrm{NC}}$-IRES-RFP). Plasmids were transfected into $293 \mathrm{~T}$ cells using deacylated polyethylenimine. Virus-containing supernatant was harvested $48 \mathrm{~h}$ later and concentrated by two rounds of ultracentrifugation. Virus titer was typically $\sim 10^{5}$ particles/ $\mu$ l. For the coinfection experiments shown in Figure $7 D$ (see below), we used a mixture of CAG-RFP and CAG-Kir-IRES-GFP retroviruses in a 1:5 ratio.

Subjects and stereotaxic surgery for retroviral delivery. Female C57BL/6 mice, $6-7$ weeks of age, were housed at four to five mice per cage in standard conditions or with two running wheels as indicated. Running wheel housing started $3 \mathrm{~d}$ before surgery and continued for the duration of the experiment. Mice were anesthetized ( $150 \mu \mathrm{g}$ ketamine $/ 15 \mu \mathrm{g}$ xylazine in $10 \mu \mathrm{l}$ of saline per gram) and virus ( $1 \mu \mathrm{l}$ at $0.15 \mu \mathrm{l} / \mathrm{min}$ ) was infused into the right dentate gyrus using sterile microcapillary calibrated pipettes (Drummond Scientific) and stereotaxic surgery [coordinates: $-2 \mathrm{~mm}$ anteroposterior $(\mathrm{AP}),-1.5 \mathrm{~mm}$ lateral $(\mathrm{L}),-1.9 \mathrm{~mm}$ ventral $(\mathrm{V})]$. A single injection site was sufficient to label a substantial number of neural precursor cells throughout the septotemporal axis of the hippocampus. In electrophysiology experiments shown in Figure 2, stereotaxic coordinates were optimized to deliver the virus to the septal $(-1.9 \mathrm{AP},-1.7 \mathrm{~L},-1.8 \mathrm{~V})$ or the temporal dentate gyrus $(-2.4 \mathrm{AP},-2.6 \mathrm{~L},-2.1 \mathrm{~V})$. Animals were killed at the indicated times $[14,18,21,35$, or $56 \mathrm{~d}$ postinfection (dpi)] for immunofluorescence or at 14,21,28, 35, or 42 dpi for electrophysiological recordings.

Bromodeoxyuridine and Arc labeling. Bromodeoxyuridine (BrdU) (Sigma-Aldrich) was dissolved in $0.9 \% \mathrm{NaCl}$ and was delivered intraperitoneally at a dose of $50 \mu \mathrm{g} / \mathrm{g}$ to mice housed under standard conditions to label dividing cells. To assess neuronal phenotype of labeled progenitors (experiments shown in Fig. 2D), mice received two injections per day over $2 \mathrm{~d}$ and were killed $21 \mathrm{~d}$ later. To measure the proportion of cells that continue to proliferate after labeling (see Fig. 3), 10 mice were injected three times in a single day (once every $6 \mathrm{~h}$ ) to label different pools of neural progenitor cells. Mice were killed 2 and $4 \mathrm{~d}$ later to assess colabeling with Ki67 (see below). For Arc immunofluorescence, running and sedentary mice were maintained in the appropriate housing conditions (sedentary or running) from 6 to 7 weeks of age and were killed $24 \mathrm{~d}$ later to match the timing of retroviral experiments done at $21 \mathrm{dpi}$.

Immunofluorescence. Immunostaining was done on 40 or $60 \mu \mathrm{m}$ freefloating coronal sections throughout the fixed brain, analyzing up to one every six sections to obtain $\geq 13 \mathrm{GFP}^{+}$or $\mathrm{BrdU}^{+}$cells to each DG region per mouse. Antibodies were applied in TBS with 3\% donkey serum and $0.25 \%$ Triton X-100. Double- or triple-labeling immunofluorescence was performed using the following primary antibodies: Arc (rabbit polyclonal; 1:500; Synaptic Systems), BrdU (rat monoclonal; 1: 200; Abcam), calbindin D-28k (rabbit polyclonal; 1:1000; Swant), doublecortin (DCX) (rabbit polyclonal; 1:750; a gift from C. Walsh, Harvard Medical School, Boston, MA), GFP (rabbit polyclonal; 1:100; Invitrogen; or chicken polyclonal; 1:500; Millipore; or chicken polyclonal; 1:500; Aves), Ki67 (rabbit monoclonal; 1:250; Vector Laboratories), NeuN (mouse monoclonal; 1:50; a gift from F. H. Gage, Salk Institute for Biological Studies, La Jolla, CA), and RFP (rabbit polyclonal; 1:200; Millipore Bioscience Research Reagents). For calbindin ( $\mathrm{Cb}$ ) immunolabeling, preincubation with methanol (15 $\mathrm{min}$ ) was included to enhance penetrability. The following corresponding secondary antibodies were used: donkey anti-mouse Cy5, donkey anti-rabbit Cy3, donkey anti-chicken Cy2 (1:250; Jackson ImmunoResearch). Although inflammation was minimal because of the thin capillary pipettes used for stereotaxic injections, we used GSA (1: 100; Vector Laboratories) and streptavidin Cy5 (1:200; Jackson ImmunoResearch) immunostaining to determine the spread of inflammation produced by the surgery and viral infection and avoid phenotypic and morphological analysis in these conditions.

For BrdU detection, DNA was denatured with $50 \%$ formamide in $2 \times$ SSC buffer at $65^{\circ} \mathrm{C}$ for $2 \mathrm{~h}$, washed in $2 \times$ SSC for $10 \mathrm{~min}$, incubated in $2 \mathrm{~N}$ $\mathrm{HCl}$ at $37^{\circ} \mathrm{C}$ for $30 \mathrm{~min}$, and washed in $0.1 \mathrm{~m}$ boric acid, $\mathrm{pH} 8.5$, for 10 min. For double labeling with the cell cycle marker Ki67 and BrdU, we used a serial protocol without formamide to avoid unspecific Ki67 labeling. Sections were first incubated with Ki67 antibody $\left(4^{\circ} \mathrm{C}\right.$; overnight), fixed with paraformaldehyde $4 \%$ for $15 \mathrm{~min}$, treated with $2 \mathrm{~N} \mathrm{HCl}$ for 20 min at room temperature, $0.1 \mathrm{~m}$ boric acid for $10 \mathrm{~min}$, washed, and followed by $\mathrm{BrdU}$ antibody incubation $\left(4^{\circ} \mathrm{C}\right.$; overnight $)$.

Anatomical definitions, confocal microscopy, and image analysis. Regions that are denominated septal and temporal in this study correspond, respectively, to sections containing the dorsal-anterior hippocampus (bregma, -0.9 to $-1.8 \mathrm{~mm}$ ) and the dorsal-posterior (intermediate) and ventral region of the hippocampus (bregma, -2.7 to -3.88 ) according to the mouse brain atlas of Paxinos and Franklin (1997).

Images were acquired using a Zeiss LSM 5 Pascal or a Zeiss LSM 510 Meta confocal microscopes (Zeiss). Only $\mathrm{GFP}^{+}$or $\mathrm{BrdU}^{+}$cells located in the subgranular zone $(<20 \mu \mathrm{m}$ thick $)$ or GCL were included in the analysis. Colocalization of fluorescent markers was assessed in threedimensional reconstructions through $Z$-stacks using multiple planes for each cell. Reconstructions included the entire cell soma for $\mathrm{Cb}, \mathrm{NeuN}$, $\mathrm{BrdU}$, and Ki67, and the cell soma plus the first $30 \mu \mathrm{m}$ of apical dendrites for DCX, as shown in the overlays depicted in Figure 2, $A$ and $B$. Images were taken at $1 \mu \mathrm{m}$ intervals using an optical slice of $0.7-1 \mu \mathrm{m}$ corresponding to an airy unit of 1 for each fluorophore [ $40 \times$ oil-immersion objective; numerical aperture (NA), 1.4; digital zoom of 2].

Arc expression was assessed from $x-y$ projections from confocal $z$-series. We calculated the proportion of DGCs expressing Arc as follows: (1) the area of the GCL was measured in septal and temporal sections and the number of $\mathrm{Arc}^{+}$cells was counted to obtain the density of $\mathrm{Arc}^{+}$cells; (2) the density of $\mathrm{NeuN}^{+}$cells per square millimeter was sampled in septal and temporal sections to correct for the angle in coronal sections and the volume of DGC somas in each region (Bayer, 1982) (17,800 \pm 900 and $12,700 \pm 900$ cells $/ \mathrm{mm}^{2}$, respectively); (3) the ratio $\mathrm{Arc}^{+}$cells/ number of $\mathrm{NeuN}^{+}$was calculated. 
For spine counts, we selected $\mathrm{GFP}^{+}$dendritic segments located in the middle third of the molecular layer. High-resolution images were acquired using a $63 \times$ objective (NA, 1.4; oil-immersion) and taking $z$-series of $40-200$ optical slices of airy unit $=1$ at $0.1 \mu \mathrm{m}$ intervals as previously described (Morgenstern et al., 2008). All dendritic spines within $2 \mu \mathrm{m}$ from the shaft were counted on projections onto a single plane. Four to 9 dendritic segments ( $40-50 \mu \mathrm{m}$ in length) were imaged per dentate gyrus region per mouse, choosing fragments at a distance $\geq 150 \mu \mathrm{m}$ from each other to avoid sampling multiple dendrites from single neurons. A similar number of fragments were chosen from the infrapyramidal and suprapyramidal blades for each area. In all cases, dendrites with high fluorescence intensity were selected to minimize counting errors because of insufficient signal. For dendritic length measurements shown in Figure $7 C-E$, images were acquired ( $40 \times$; NA, 1.3; oil-immersion) from $60-\mu \mathrm{m}$-thick sections taking $z$-series including $35-50$ optical slices, airy unit $=1$ at $0.8 \mu \mathrm{m}$ intervals. The Kir-IRES-GFP retrovirus rendered low fluorescence intensity that was insufficient for dendritic measurements. To solve that problem, dendritic trees of Kir-expressing neurons were characterized after coinfection with two retroviruses (Fig. 7D): a Kir-IRES-GFP construct (which allowed identifying the soma of Kir neurons) and a CAGRFP construct (which rendered high fluorescence in soma and processes). Dendritic length was measured from projections of three-dimensional reconstructions onto a single plane using Zeiss LSM image Browser software.

Three-dimensional reconstructions of dendrites quantified in Figure $1 D$ were performed in $400-\mu \mathrm{m}$-thick vibratome coronal sections. Slices were fixed ( $4 \%$ paraformaldehyde) and mounted. Images were taken using a Zeiss LSM 710 NLO multiphoton microscope, a $20 \times$ waterimmersion objective (NA, 1.0), and a Chameleon Ultra II pulsed laser (Coherent). Series of 200-400 optical slices were acquired at $1 \mu \mathrm{m}$ intervals. Dendritic length was measured from the three dimensional reconstructions using the ZEN 2009 software (Carl Zeiss).

Electrophysiology. Mice were anesthetized and decapitated at 14, 21, 28, 35 , or $42 \mathrm{dpi}$ as indicated. Brains were removed into a chilled solution containing the following (in $\mathrm{mm}$ ): 110 choline- $\mathrm{Cl}^{-}, 2.5 \mathrm{KCl}, 2.0$ $\mathrm{NaH}_{2} \mathrm{PO}_{4}, 25 \mathrm{NaHCO}_{3}, 0.5 \mathrm{CaCl}_{2}, 7 \mathrm{MgCl}_{2}, 20$ dextrose, $1.3 \mathrm{Na}^{+}$ascorbate, $0.6 \mathrm{Na}^{+}$-pyruvate, and 4 kynurenic acid. Horizontal slices (400 $\mu \mathrm{m}$ thick) were cut in a vibratome (Leica VT1200 S) and transferred to a chamber containing the following (in $\mathrm{mm}$ ): $125 \mathrm{NaCl}, 2.5 \mathrm{KCl}, 2$ $\mathrm{NaH}_{2} \mathrm{PO}_{4}, 25 \mathrm{NaHCO}_{3}, 2 \mathrm{CaCl}_{2}, 1.3 \mathrm{MgCl}_{2}, 1.3 \mathrm{Na}^{+}$-ascorbate, 3.1 $\mathrm{Na}^{+}$-pyruvate, and 10 dextrose $(315 \mathrm{mOsm})$. Slices were bubbled with $95 \% \mathrm{O}_{2} / 5 \% \mathrm{CO}_{2}$ and maintained at $30^{\circ} \mathrm{C}$ for $>1 \mathrm{~h}$ before experiments started. Whole-cell recordings were performed at $23 \pm 2^{\circ} \mathrm{C}$ using microelectrodes (4-6 M $\Omega$ ) pulled from borosilicate glass (KG-33; King Precision Glass) and filled with the following (in $\mathrm{mM}$ ): $120 \mathrm{~K}$-gluconate, 20 $\mathrm{KCl}, 5 \mathrm{NaCl}, 4 \mathrm{MgCl}_{2}, 0.1$ EGTA, 10 HEPES, 4 Tris-ATP, 0.3 Tris-GTP, 10 phosphocreatine, Alexa Fluor 594 ( $5 \mu \mathrm{g} / \mathrm{ml}$; Invitrogen), $\mathrm{pH} 7.3$, and 290 mOsm. For mIPSC recordings, electrodes were filled with a similar solution, except for the following (in mM): $19 \mathrm{~K}$-gluconate, $121 \mathrm{KCl}, 5$ $\mathrm{NaCl}, 4 \mathrm{MgCl}_{2}, 0.1 \mathrm{EGTA}$. Recordings were obtained using an Axopatch 200B amplifier (Molecular Devices), digitized (Digidata 1322A), and acquired at $20 \mathrm{kHz}$ onto a personal computer using the pClamp 9 software (Molecular Devices). Membrane capacitance and input resistance were obtained from current traces evoked by a hyperpolarizing step of $10 \mathrm{mV}$. In current-clamp recordings, the resting membrane potential was kept at $-70 \mathrm{mV}$ by passing a holding current. The threshold current for spiking was assessed by successive depolarizing current steps ( $5 \mathrm{pA} ; 200 \mathrm{~ms})$ to drive the membrane potential from resting to $0 \mathrm{mV}$. Voltage-dependent $\mathrm{Na}^{+}$and $\mathrm{K}^{+}$currents were measured after leak subtraction using a $\mathrm{p} / 6$ protocol and automatic detection of the fast inward peak and the late outward plateau. Kir currents were measured as the steady-state inward deflection in response to hyperpolarizing voltage steps (from -130 to $-90 \mathrm{mV}$ ). Kir conductance was calculated after subtracting the leak conductance measured between -60 and $-40 \mathrm{mV}$, where Kir channels are closed (Mongiat et al., 2009). Spontaneous postsynaptic excitatory currents (sEPSCs) were recorded at a holding potential of $-70 \mathrm{mV}$, in the presence of picrotoxin $(100 \mu \mathrm{M})$ and (2S)-3-([(1S)-1-(3,4-dichlorophenyl)ethyl]amino-2hydroxypropyl)(phenylmethyl)phosphinic acid (CGP55845) (100 nM). Miniature EPSCs (mEPSCs) and miniature IPSCs (mIPSCs) were recorded in the presence tetrodotoxin $(0.5 \mu \mathrm{M})$ and CGP55845 (100 nM), and picro-
A

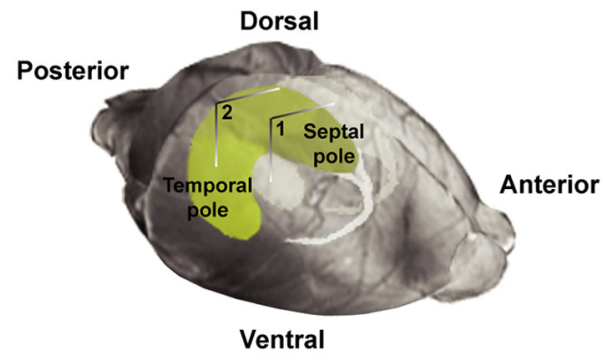

B Septal (1) Temporal (2)
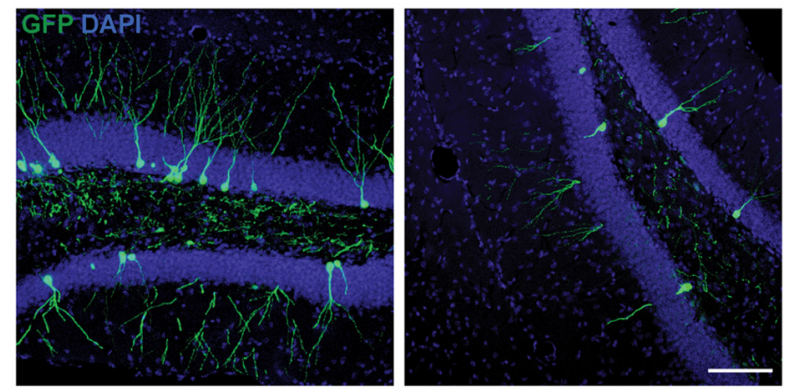

C
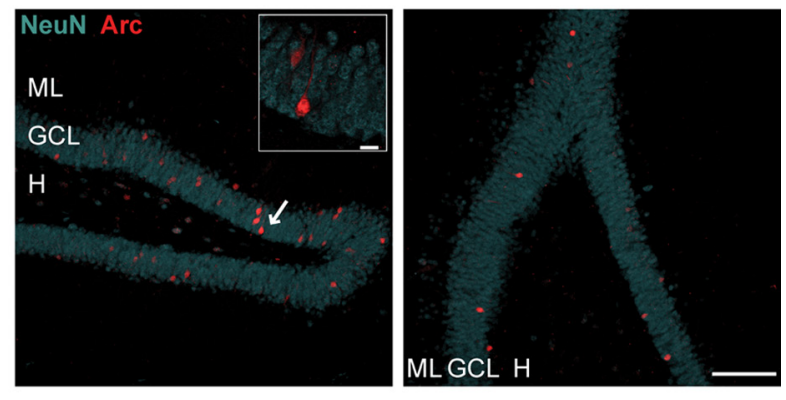

D

E
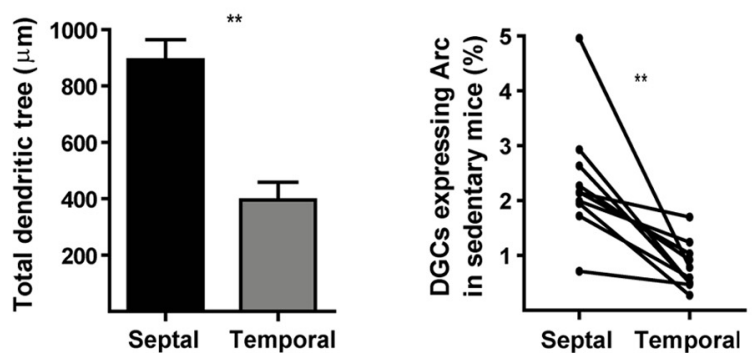

Figure 1. Adult-born DGCs in the septal and temporal hippocampus. Differences in morphology match local network activity. $\boldsymbol{A}$, Schematic view of the mouse brain depicting the three-dimensional anatomy of the hippocampus and two coronal planes, examples of the septal (1) and temporal (2) areas analyzed in this work. $\boldsymbol{B}$, Retrovirally labeled neurons at $21 \mathrm{dpi}$. Typical examples of newborn DGCs in the septal (1) and temporal (2) dentate gyrus of the same mouse, with the planes oriented as shown in $A$. Note that GFP ${ }^{+}$neurons (green) in the septal section display more elaborated dendritic trees spreading the entire molecular layer, whereas those in the temporal section appear more immature. DAPI nuclear labeling is shown in blue. C, Representative images of Arc expression (red) in the septal (left panel) and temporal (right panel) dentate gyrus of the same mouse. ML, Molecular layer; $\mathrm{GCL}$, granule cell layer; $\mathrm{H}$, hilus. NeuN immunofluorescence is shown in blue. Inset, Higher magnification of a DGC expressing Arc, pointed by the arrow. Shown is a single plane overlay of Arc and NeuN labeling. $\boldsymbol{D}$, Quantitative analysis of the total dendritic length of newborn DGCs at 21 dpi septal and temporal hippocampus after two-photon imaging of $400-\mu \mathrm{m}$-thick coronal sections. ${ }^{* *} p<$ 0.0015 , Mann-Whitney test. The bars represent mean \pm SEM with $n=7$ septal neurons obtained from three mice and $n=12$ temporal neurons from two mice. $\boldsymbol{E}$, Arc expression in the $G C L$ analyzed by pairing septal and temporal sections, where each pair of dots joined by a line represents data obtained from the same mice. ${ }^{* *} p=0.002$, Wilcoxon's test; $n=10$ sedentary mice. Scale bars: $100 \mu \mathrm{m}$; inset, $10 \mu \mathrm{m}$. 
A Septal
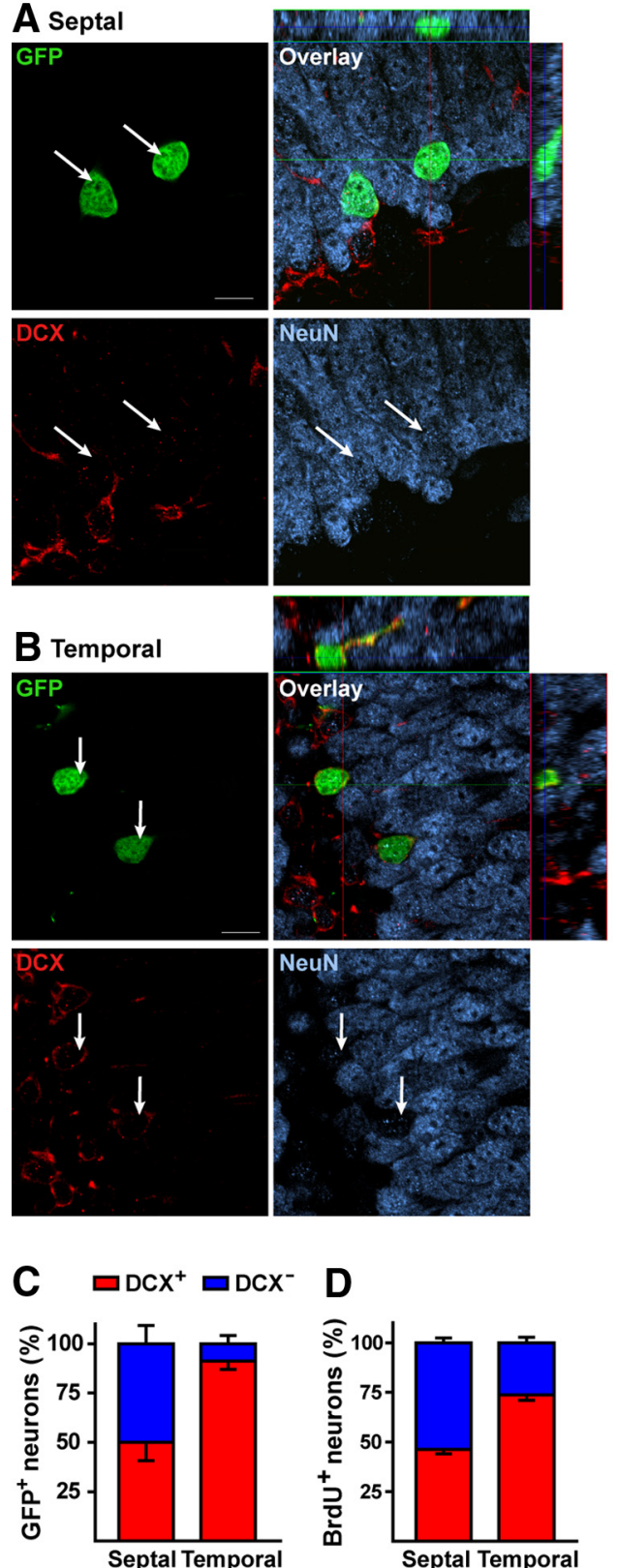
Hippocampus region

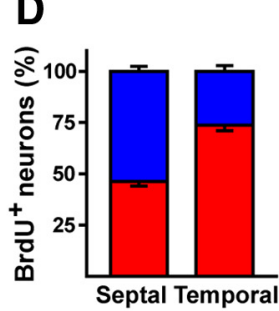

E

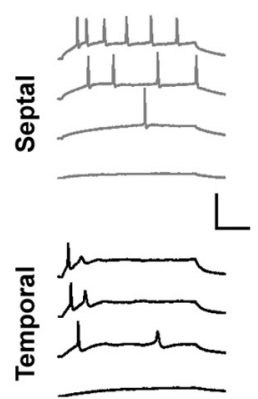

G

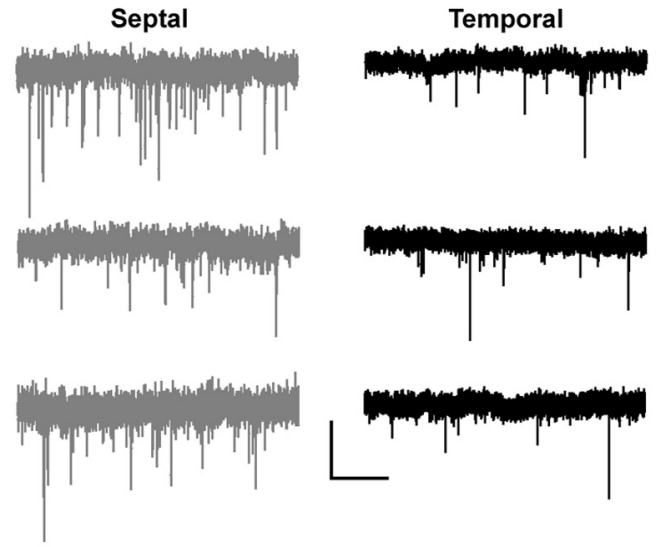

H

Figure 2. Three-week-old neurons achieve distinct degrees of maturation that depend on their septotemporal localization. $A, B$, Examples of retrovirally labeled newborn DGCs expressing NeuN but not DCX in a septal section $(\boldsymbol{A})$ and neurons expressing DCX in a temporal section of the same brain (B). Images display single optical sections of GFP (green), DCX (red), and NeuN (blue) channels shown separately. Their overlays and orthogonal projections are shown on the right top panel of each example. Scale bars, $10 \mu \mathrm{m}$. Note that neurons lacking DCX exhibited stronger NeuN expression, suggesting a more advanced degree of maturation in the septal region. C, Proportion of GFP ${ }^{+}$neurons expressing DCX $\left(D C X^{+}\right)$. All DCX ${ }^{-}$neurons expressed NeuN. The bars denote mean \pm SEM $\left(n=7\right.$ mice, with $191 \mathrm{GFP}^{+}$neurons in the septal region and $174 \mathrm{GFP}^{+}$neurons in the temporal region; $p=0.004$, Mann-Whitney test, DCX septal vs temporal). $\boldsymbol{D}$, Proportion of BrdU ${ }^{+}$neurons expressing DCX. The colored bars show mean \pm SEM obtained from five mice with $n=85$ neurons (septal region) and $n=122$ neurons (temporal region; $p=0.008$, Mann-Whitney test). $\boldsymbol{E}$, Representative traces of whole-cell current-clamp recordings in septal (top) and temporal (bottom) GFP ${ }^{+}$neurons. Spiking was elicited by depolarizing current steps of increasing amplitude (step, $5 \mathrm{pA}$ ). Each panel shows a subset of four representative traces elicited by 10,30,50, and $70 \mathrm{pA}$ steps (from bottom to top). Calibration: $100 \mathrm{mV}, 50 \mathrm{~ms}$. $F$, Number of action potentials elicited by injected current steps $\left(V_{\text {resting }}\right.$ kept at $-70 \mathrm{mV}$ ). Data depict mean \pm SEM, with $n=15$ (septal) and 14 (temporal) new DGCs. G, Voltage-clamp recordings of sEPSCs recorded at $-70 \mathrm{mV}$ in presence of $100 \mu \mathrm{m}$ picrotoxin. Traces correspond to six different 21 dpi neurons located in the septal (left) and temporal regions of the dentate gyrus (right). Calibration: $5 \mathrm{pA}, 2 \mathrm{~s}$. $\boldsymbol{H}$, Septal neurons exhibit higher sEPSC frequency. ${ }^{*} p=0.032$ for $t$ test, with $n=14$ (septal) and 12 (temporal) GFP ${ }^{+}$DGCS. I, Cumulative histogram displaying similar sEPSC amplitudes for the recordings analyzed in $\boldsymbol{H}$.

toxin (100 $\mu \mathrm{m}$; for mEPSCs) or kynurenic acid (4 mM; for mIPSCs). Criteria to include cells in the analysis were colabeling with Alexa Fluor 594 or visual confirmation of GFP in the pipette tip, and absolute leak current $<50 \mathrm{pA}$ at $-70 \mathrm{mV}$. Series resistance was typically $10-20 \mathrm{M} \Omega$, and experiments were discarded if $>30 \mathrm{M} \Omega$.

Statistical analysis. Statistics used throughout the manuscript are described in the figure legends and in the text. All comparisons between neu- ronal populations involving the septal and temporal hippocampus of the same mice were done using paired analysis. When data met normality tests (Gaussian distribution and equal variance), two-tailed $t$ tests or ANOVA were used, as indicated. In cases in which data did not meet normality criteria, nonparametric tests were used as follows: Mann-Whitney test for independent comparisons, Wilcoxon's test for simple paired comparisons, Kruskal-Wallis test for multiple comparisons, and Dunn's or Conover's test as post hoc analysis. 


\section{Results}

Adult-born DGCs from the same cohort exhibit different degrees of maturation along the septotemporal axis of the hippocampus

The anatomical and functional gradients along the hippocampal axis might entail differences in neuronal development. We first investigated possible differences for immature neurons of the same cohort between the septal and temporal region of the hippocampus of sedentary mice. To address this question, dividing neural progenitor cells were labeled in the dentate gyrus of 6- to 7-week-old mice by injecting a retrovirus that expresses GFP (Laplagne et al., 2006). GFP ${ }^{+}$neurons were characterized 21 dpi, a critical neuronal age that is coincident with a high rate of glutamatergic synaptogenesis (Espósito et al., 2005; Ge et al., 2006; Zhao et al., 2006). Interestingly, new DGCs developing in the septal hippocampus displayed a larger and more elaborated dendritic tree than neurons from the same cohort developing in the temporal region (Fig. 1A,B). Dendritic length of 21 dpi neurons was then measured using two-photon microscopy in $400-\mu \mathrm{m}$ thick sections to obtain a complete reconstruction of the dendritic tree. A significantly longer dendritic tree was visualized in newborn cells of the septal region compared with those developing in the temporal area (Fig. 1D). Since network activity is different along the longitudinal axis of the hippocampus under basal conditions (Jung et al., 1994; Snyder et al., 2009a,b), we assessed the expression of the immediate early gene Arc as a sensor for neuronal activity in the entire GCL of the septal and temporal dentate gyrus (Chawla et al., 2005; Ramirez-Amaya et al., 2006; Vazdarjanova et al., 2006; Kee et al., 2007). The overall number of active DGCs was low, consistent with the sparse coding of the dentate gyrus (Chawla et al., 2005; Leutgeb et al., 2007); yet substantial differences between hippocampal areas were observed (Fig. 1C,E). Arc immunofluorescence was detected in $\sim 2.7 \%$ of septal DGCs and $<1 \%$ of temporal DGCs, which reflects a higher level of basal activity in the septal dentate gyrus, in agreement with recent works (Snyder et al., 2009a,b). Remarkably, the higher proportion of Arc-expressing $\left(\mathrm{Arc}^{+}\right)$neurons in the septal area was observed in all tested mice, highlighting a very robust difference in the levels of network activity in the longitudinal axis of the dentate gyrus. These results suggest that young DGCs from the same cohort exhibit a highly elaborated morphology in the more active region (septal) of the adult dentate gyrus.

To assess whether the distinct morphologies described above reflect true differences in the degree of maturation of newborn DGCs in local environments of the hippocampus, we measured the proportion of $\mathrm{GFP}^{+}$neurons expressing DCX, a microtubule-associated protein present during neuronal migration that is commonly used to label immature neurons (Espósito et al., 2005). NeuN was used as a constitutive neuronal marker and counterstain for the GCL. At 21 dpi, DCX expression was observed in $\sim 50 \%$ of $\mathrm{GFP}^{+}$neurons at the septal dentate gyrus but in $>90 \%$ of newborn DGCs at the temporal region (Fig. $2 A-C$ ). The remaining $\mathrm{GFP}^{+}$cells expressed only NeuN $\left(\mathrm{DCX}^{-}\right)$, which confirms their neuronal phenotype. Therefore, migration has been completed in $\sim 50 \%$ of newborn DGCs in the septal hippocampus, but in only $\sim 10 \%$ of DGCs developing in the temporal region. Septotemporal differences in neuronal maturity at 3 weeks were further confirmed by labeling dividing progenitor cells by systemic delivery of BrdU, a noninvasive labeling technique (Fig. $2 D$ ). This experimental condition rendered a similar pattern to that obtained with retroviral labeling, with higher DCX expression in newborn cells of the temporal hippocampus. Together, these results demonstrate that DGCs
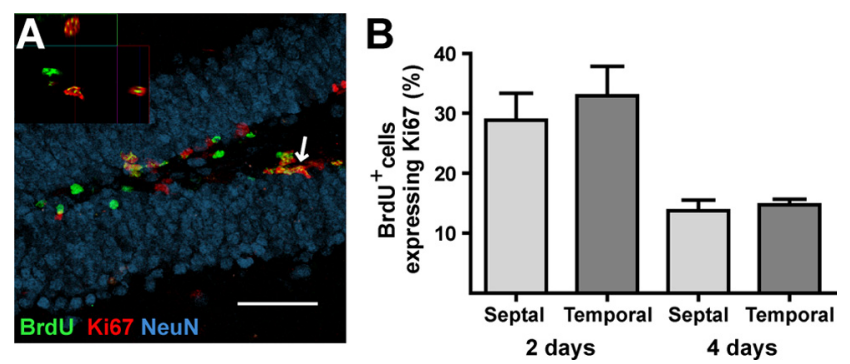

Figure 3. Neural progenitor cells of the septal and temporal hippocampus display similar timing to exit the cell cycle. $A$, Overview of the $\mathrm{GCL}$ of the septal dentate gyrus labeled with NeuN (blue) depicting subgranular labeling for BrdU (green) and the cell cycle marker Ki67 (red), shown $4 \mathrm{~d}$ after BrdU injection. Note the clusters of cells that continue to divide (yellow, double BrdU and Ki67 labeling). Scale bar, $50 \mu \mathrm{m}$. Inset, Orthogonal projection of the cell pointed by the arrow, displaying double BrdU and Ki67 labeling. B, Proportion of BrdU ${ }^{+}$cells labeled with Ki67. No significant differences were found for septal versus temporal areas ( $p>0.05$, twoway ANOVA). The bars represent mean \pm SEM, with $n=5$ mice at each time point $(>500$ $\mathrm{BrdU}^{+}$cells for each region and at each time point).

born at the septal pole of the hippocampus achieve a more mature neuronal phenotype in 3 weeks than those generated at the temporal region.

To determine whether the observed differences in the pattern of expression of neuronal markers also implicate neuronal physiology and connectivity, electrophysiological properties of adult-born DGCs were studied at $21 \mathrm{dpi}$. Whole-cell recordings were performed in $\mathrm{GFP}^{+}$neurons in acute slices obtained from the septal and temporal areas of the hippocampus. Recordings from GFP ${ }^{+}$DGCs of the septal hippocampus revealed a lower input resistance $\left(R_{\text {input }}\right)$ than those from temporal GFP ${ }^{+} \mathrm{DGCs}\left(R_{\text {input }}=636 \pm 94 \mathrm{M} \Omega, n=\right.$ 25 for septal; $1900 \pm 420 \mathrm{M} \Omega, n=24$ for temporal; $p=0.007, t$ test), typical of a more advanced degree of maturation. New DGCs of the septal region also showed more mature firing properties as seen by the larger amplitude of voltage-gated $\mathrm{Na}^{+}$and $\mathrm{K}^{+}$currents and the repetitive spiking elicited by depolarizing current steps (Fig. 2E,F). The maximum $\mathrm{Na}^{+}$and $\mathrm{K}^{+}$conductances were $344 \pm 31$ and $53.6 \pm 4.9 \mathrm{nS}$ ( $n=16$ for septal), and $148 \pm 29$ and $27.1 \pm 5.3 \mathrm{nS}(n=14$ for temporal DGCs; $p<$ 0.0001 for $\mathrm{Na}^{+} ; p=0.001$ for $\mathrm{K}^{+}, t$ test). Finally, the frequency but not the amplitude of sEPSCs was significantly higher in septal newborn DGCs $(\sim 1$ vs $\sim 0.2 \mathrm{~Hz})$, reflecting a higher degree of afferent glutamatergic connectivity than the temporal newborn DGCs (Fig. 2G-I). Therefore, all of the assessed functional properties indicate that new DGCs developing at the septal pole of the adult hippocampus are ready to participate in information processing at a time when new DGCs of the same cohort in the temporal region are still highly immature and poorly integrated in the preexisting circuitry.

\section{Slower rate of neuronal maturation in the temporal dentate gyrus}

The differences in the degree of neuronal maturation described above could be compatible with the following scenarios: (1) progenitor cells in the temporal dentate gyrus continue to proliferate and exit the cell cycle at a later time, beginning their maturation only after a certain delay; (2) neurons developing in the temporal region are stalled at immature stages and fail to reach a fully mature phenotype; (3) maturation occurs at different rates along the longitudinal axis of the adult hippocampus. A series of experiments were performed to discriminate between these possibilities.

We first analyzed possible septotemporal differences in the time at which neural progenitor cells abandon the cell cycle. To 
address this issue, we injected BrdU for $1 \mathrm{~d}$ to label dividing cells of the subgranular zone, and evaluated the proportion of those cells that continue to proliferate at later times. The cell cycle marker Ki67 was used to identify cells that continue to divide 2 and $4 \mathrm{~d}$ after BrdU injection. Consistent with previous observations, $\sim 30 \%$ of the dividing cells were still proliferating $\left(\mathrm{BrdU}^{+} / \mathrm{Ki} 7^{+}\right)$in the whole dentate gyrus $2 \mathrm{~d}$ after BrdU injection. Moreover, the density of new cells $\left(\mathrm{BrdU}^{+}\right)$at $2 \mathrm{~d}$ was higher in the septal dentate gyrus by $33.1 \pm 14.0 \%$ when compared with the temporal region $(p=0.032$, paired $t$ test $)$ (Dayer et al., 2003; Snyder et al., 2009a,b; Jinno, 2011). However, no differences in the proportion of cells that were still proliferating $\left(\mathrm{BrdU}^{+} / \mathrm{Ki} 67^{+}\right)$were found at 2 or $4 \mathrm{~d}$ after BrdU injections in the septal versus temporal regions ( $~ 30$ and $\sim 15 \%$ ) (Fig. 3). These results indicate that both pools of neural progenitor cells exit the cell cycle at the same time. Therefore, the observed differences in the development of septal and temporal DGCs are not attributable to a dissimilar onset of neuronal differentiation.

We then compared morphology and expression of neuronal markers of newborn DGCs in the septal and temporal hippocampus at 21 and 56 dpi to address whether new DGCs of the temporal region fail to reach a fully mature phenotype. For these experiments, we selected $56 \mathrm{dpi}$ as the time when adult-born DGCs have already completed their maturation (Espósito et al., 2005; Laplagne et al., 2006; Zhao et al., 2006). Despite the fact that 21 dpi neurons showed profound septotemporal differences, all new neurons displayed similar features by $56 \mathrm{dpi}$ (Fig. 4A-D). Since the highest rate of dendritic spine formation in adult-born DGCs occurs by the third week, we measured spine density as a high-resolution parameter that accurately reflects the degree of maturation. By 21 dpi, DGCs developing at the septal hippocampus displayed a rather mature phenotype with spiny dendrites $(\sim 1.5$ spines $/ \mu \mathrm{m})$ spanning the entire molecular layer (Fig. $4 A, E, G)$. At 56 dpi, new neurons in this area showed a marked increase in spine density ( $\sim 2.5$ spines/ $\mu \mathrm{m}$ ) but maintained their overall mature features (Fig. $4 C, F, G$ ). In striking contrast, 21 dpi neurons in the temporal region displayed simple thin dendrites restricted to the middle molecular layer, dendritic varicosities, low spine density $(\sim 0.8$ spines $/ \mu \mathrm{m})$, and basal dendrites projecting to the hilus, all typical properties of an immature dendritic tree (Figs. $4 B, E, G, 1 D$ ) (Jones et al., 2003). These results are in agreement with the electrophysiological recordings described above displaying immature intrinsic properties and reduced glutamatergic connectivity onto tempo-
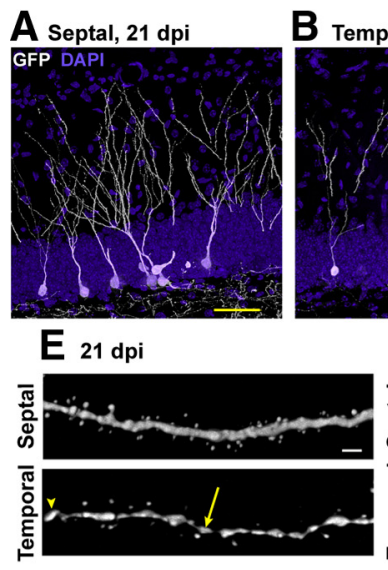

H

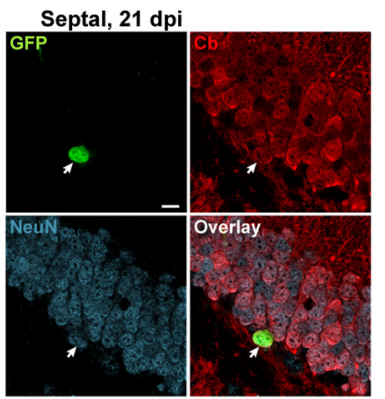

1

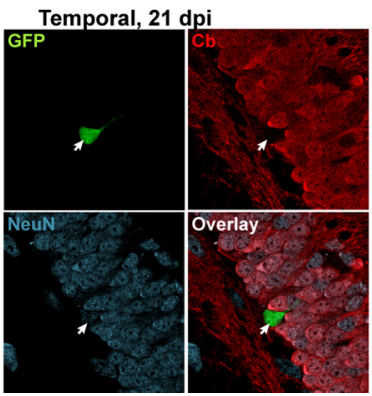

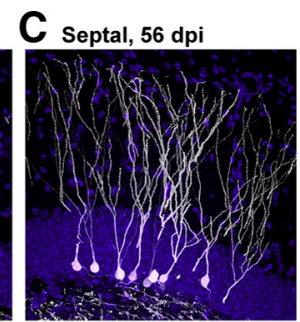

Demporal, $56 \mathrm{dpi}$

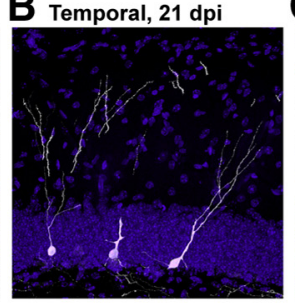

$F_{56} \mathrm{dpi}$

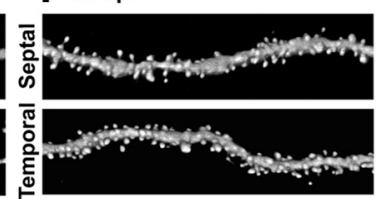

G

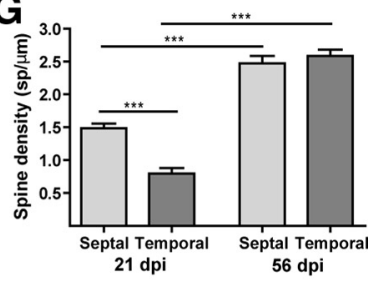

J

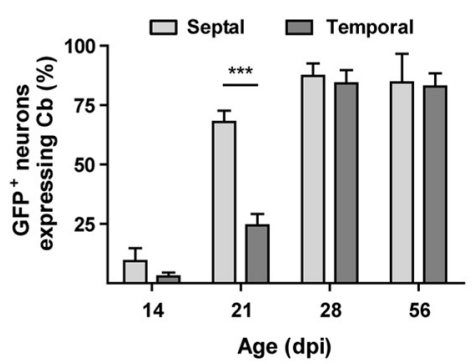

K

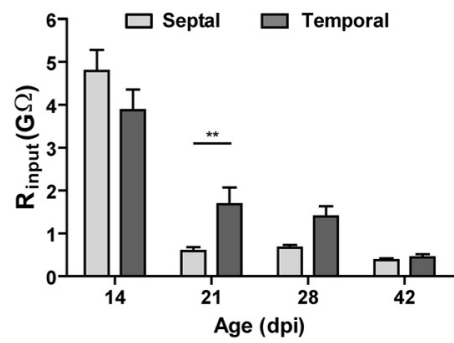

Figure 4. Slow rate of maturation for adult-born DGCs in the temporal dentate gyrus. $A-D$, Representative confocal images of newly generated DGCs in the septal and temporal dentate gyrus obtained at 21 and $56 \mathrm{dpi}$. GFP ${ }^{+}$neurons (white) are shown in a DAPI (blue) background labeling the GCL. Scale bar, $50 \mu \mathrm{m}$. Note that the striking septotemporal differences seen at $21 \mathrm{dpi}$ are no longer found at $56 \mathrm{dpi}$. $\boldsymbol{E}, \boldsymbol{F}$, Example dendritic segments obtained from septal and temporal newborn cells of the same mice, at the indicated times. Scale bar, $2 \mu \mathrm{m}$. Note the presence of dendritic varicosities (arrow) and the dendrite end in the middle molecular layer (arrowhead) found in the temporal region at $21 \mathrm{dpi}$, typical features of an immature neuron. $\mathbf{G}$, Dendritic spine counts reveal low density in temporal DGCs at $21 \mathrm{dpi} . n=58$ total fragments (septal plus temporal) taken from five mice at $21 \mathrm{dpi}$, and 26 fragments from three mice at $56 \mathrm{dpi}$. ${ }^{* * *} p<0.001$, ANOVA with Bonferroni's post hoc test. $\boldsymbol{H}, \boldsymbol{I}$, Example of 21 dpi neurons (green) obtained from the same mouse depicting $\mathrm{a} \mathrm{Cb}^{+}$(red) and $\mathrm{NeuN}^{+}$(blue) expression in the septal dentate gyrus $(\boldsymbol{H})$ and $\mathrm{a} \mathrm{Cb}^{-}$ with weak NeuN expression in the temporal region $(\boldsymbol{I})$. Images show single optical sections for each channel and their overlay. Scale bar, $10 \mu \mathrm{m} . J$, Cb expression in developing DGCs. $n=4$ mice and 257 neurons ( $14 \mathrm{dpi}$ ), 8 mice and 444 neurons ( $21 \mathrm{dpi}$ ), 5 mice and 279 neurons ( $28 \mathrm{dpi}$ ), 4 mice and 183 neurons ( $56 \mathrm{dpi}$ ). ${ }^{* *} p<0.001$, ANOVA with Bonferroni's post hoc test. $\boldsymbol{K}$, Input resistance in GFP ${ }^{+}$neurons recorded in whole cell as a function of age in the septal and temporal dentate gyrus. ${ }^{* *} p<0.01$, two-way ANOVA followed by Bonferroni's post hoc test. All bars represent mean \pm SEM.

ral DGCs (Fig. 2). However, neuronal maturation in the temporal region displayed a clear progress by $56 \mathrm{dpi}$, reflected by both a qualitative change in morphology and a significant increase in spine density (Fig. 4D, F, G). Therefore, by $56 \mathrm{dpi}$, all new neurons were equally and completely mature, displaying a round soma, conspicuous axonal fibers extending toward the hilus, and dendrites spanning the molecular layer with a high spine density characteristic of mature DGCs (Trommald and Hulleberg, 1997; Zhao et al., 2006). These observations argue against the possibility that temporal newborn cells fail to reach complete maturation. 
A Septal

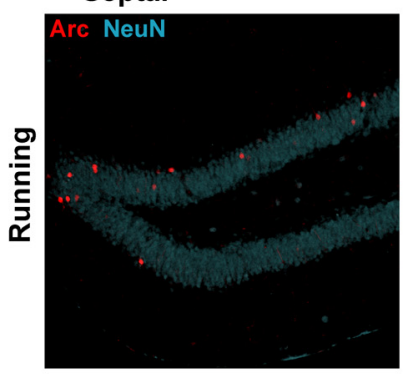

C
Temporal

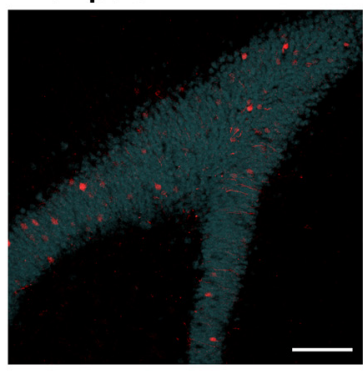

D Septal
B

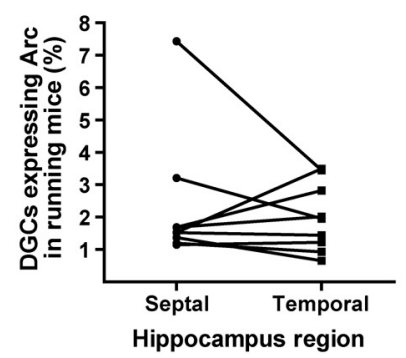

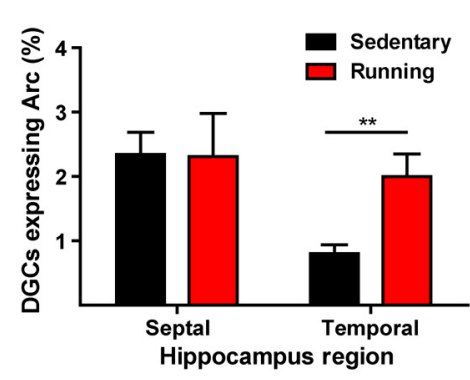

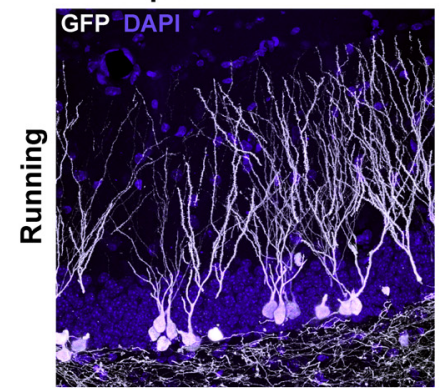

Temporal

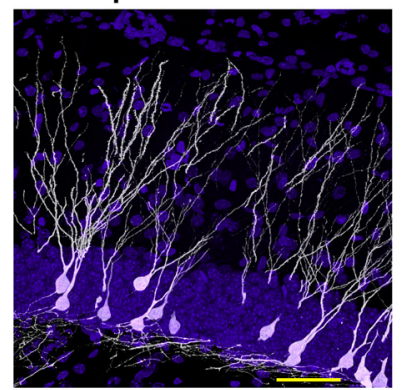

E Septal

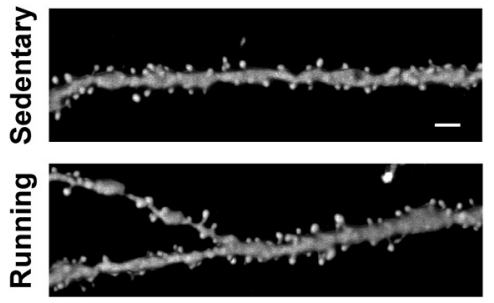

Temporal
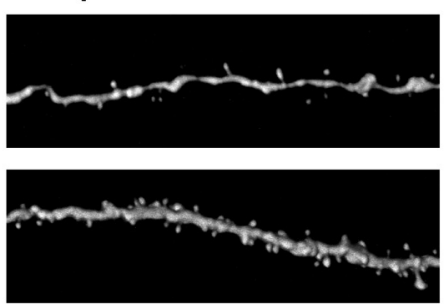

F

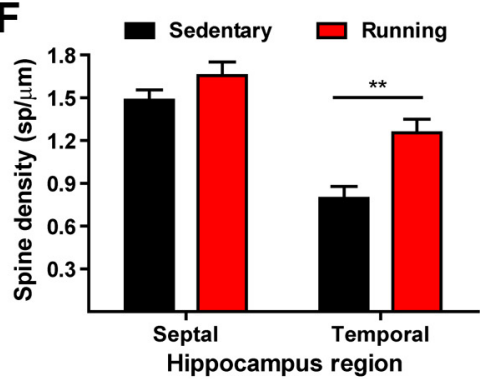

H

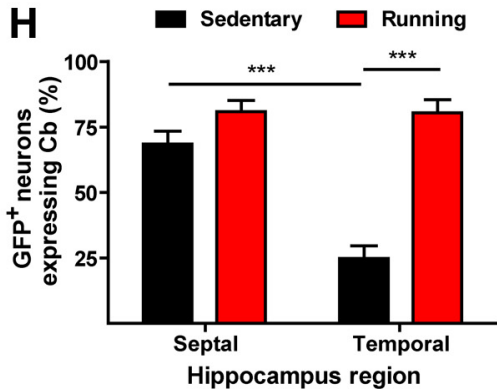

Figure 5. Running increases network activity in the temporal GCL and accelerates neuronal maturation. $A$, Representative images of Arc expression (red) in the septal and temporal dentate gyrus of running mice. NeuN immunofluorescence is shown in blue. Note the similar level of Arc expression (red) in the septal and temporal GCL. Scale bar, $100 \mu \mathrm{m}$. B, Paired analysis of Arc expression in sections obtained from the same running mice. No significant differences were found ( $p=0.73$, Wilcoxon's test; $n=9)$. C, Running increases Arc expression in the temporal GCL. The bars combine the data shown in Figures $1 E$ and $5 B .{ }^{* *} p<0.01$, Kruskal-Wallis followed by post hoc Conover's test. $\boldsymbol{D}$, Examples of newborn DGCs (white) at 21 dpi in the septal and temporal dentate gyrus of the same running mouse. Note the similar degree of maturation in both regions. Sections were counterstained with DAPI (blue). Scale bar, $50 \mu \mathrm{m}$. $E$, Dendritic segments of $21 \mathrm{dpi} \mathrm{neurons} \mathrm{from} \mathrm{the} \mathrm{septal}$ and temporal dentate gyrus in sedentary and running mice, as indicated. Scale bar, $2 \mu \mathrm{m}$. $\boldsymbol{F}$, Running increases spine density in newborn DGCs of the temporal dentate gyrus. Spine count analysis in 21 dpi neurons, with $n=45$ total (septal plus temporal) fragments taken from four running mice. Data for sedentary mice are the same as that shown in Figure 4G. ** $p<0.01$, ANOVA with Bonferroni's post hoc test. G, Newborn DGCs (green) at 21 dpi in the temporal dentate gyrus of a running mouse expressing both Cb (red) and NeuN (blue). Images are single optical planes for each channel and their overlay. Scale bar, $10 \mu \mathrm{m}$. $\boldsymbol{H}$, Running increases Cb expression in 21 dpi neurons of the temporal hippocampus. The bars show the proportion of GFP ${ }^{+}$cells that display $\mathrm{Cb}$ expression, with $n=462$ neurons from eight running mice compared with data shown in Figure $4 \mathrm{~J}$ for sedentary mice. ${ }^{* * *} p<0.001$, two-way ANOVA with Bonferroni's post hoc test. All bars denote mean \pm SEM.

Finally, to strengthen the finding that new septal DGCs mature faster than new temporal DGCs, we monitored two parameters that reflect the phenotypic and physiological aspects of neuronal development. We first studied the time course of $\mathrm{Cb}$ expression, which is a specific neuronal marker commonly used to identify the latest stages of development in adult-born DGCs (Zhao et al., 2008). Although Cb immunoreactivity was detected in few neurons $(<10 \%)$ in both the septal and temporal dentate gyrus at $14 \mathrm{dpi}$, a striking increase in $\mathrm{Cb}$ expression to almost plateau levels occurred only in the septal DGCs at 21 dpi (Fig. $4 H-J)$. By 28 dpi, most DGCs displayed Cb expression regardless of their longitudinal position, suggesting that all new neurons have entered their latest stages of maturation. However, although $\mathrm{Cb}$ is usually considered as a mature neuronal marker, it seems to 
be expressed in developing DGCs that have not yet reached functional and morphological maturity (Espósito et al., 2005; Piatti et al., 2006; Toni et al., 2008; Zhao et al., 2008). Since membrane properties display reliable changes as neurons progress along different stages of maturation (Espósito et al., 2005; Piatti et al., 2006), we compared $R_{\text {input }}$ between new DGCs developing in the septal and temporal hippocampus (Fig. $4 K$ ). Although septal DGCs displayed an abrupt decline in $R_{\text {input }}$ between 14 and $21 \mathrm{dpi}$, temporal DGCs exhibited a more gradual decrease in the examined interval. Notably, $R_{\text {input }}$ measurements revealed striking differences at $28 \mathrm{dpi}(667 \pm 67 \mathrm{M} \Omega$ for septal and $1400 \pm 240 \mathrm{M} \Omega$ for temporal DGCs), whereas $\mathrm{Cb}$ expression had reached similar values in septal and temporal DGCs (Fig. $4 J)$. $R_{\text {input }}$ values typical of mature DGCs were found in new neurons of both regions by $42 \mathrm{dpi}(379 \pm 44 \mathrm{M} \Omega$ for septal and $440 \pm 78 \mathrm{M} \Omega$ for temporal DGCs). These results highlight the lengthened interval during which newborn DGCs of the temporal hippocampus maintain immature functional properties. Based on marker expression, morphology, electrophysiological properties, and connectivity, we conclude that neuronal maturation progresses at a faster rate at the septal compared with the temporal region of the adult dentate gyrus.

\section{Enhanced network activity by voluntary} exercise accelerates neuronal maturation To investigate how neuronal activity of the local environment impinges in the rate of neuronal maturation, we sought for physiological means to modulate circuit activity. Network activity in the hippocampus is coupled to voluntary locomotion (Vanderwolf, 1969; Foster et al., 1989). For instance, the firing rate increases with running speed in different longitudinal regions of the hippocampus (Czurkó et al., 1999; Maurer et al., 2005).

We therefore explored the effects of long-term running on the level of basal activity in the GCL. Interestingly, running did not modify the proportion of active $\left(\mathrm{Arc}^{+}\right)$DGCs in the septal region, but, in contrast, it did produce a substantial increase $(>150 \%)$ in the proportion of $\mathrm{Arc}^{+}$DGCs in the temporal region (Fig. 5A,B). This specific change in neuronal activity by running equalized the proportion of active DGCs in the septal and temporal hippocampus (Fig. 5C).

We then compared the degree of maturation of 3-week-old neurons in the septal and temporal hippocampus of sedentary and running mice. We first observed a conspicuous increase in the number of $\mathrm{GFP}^{+}$neurons (Fig. 5D), consistent with the well known enhancing effect of voluntary exercise on the rate of progenitor cell proliferation (van Praag et al., 1999). Most strikingly, newborn DGCs at 21 dpi displayed mature features in both hip-
A

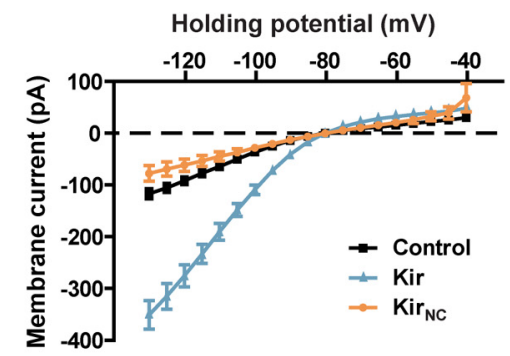

B

C

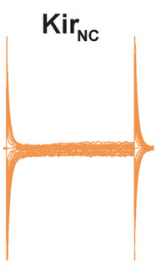

D

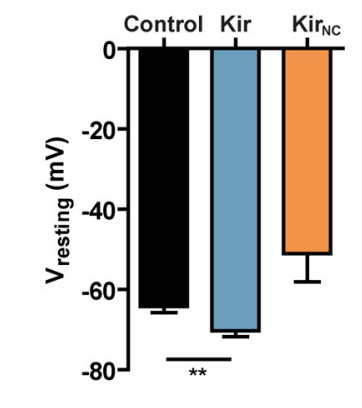

$\mathbf{F}$

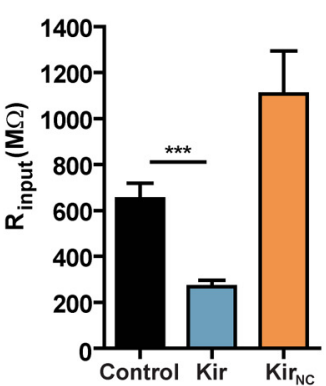

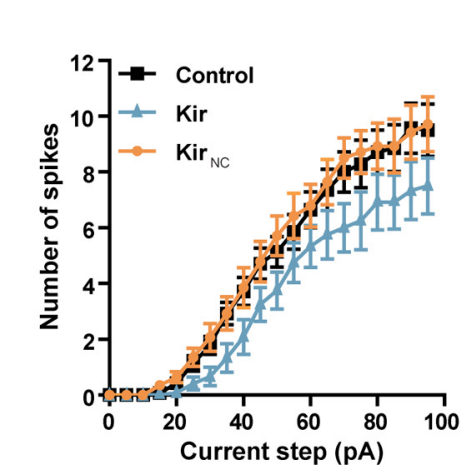

Figure 6. Overexpression of Kir2.1 reduces the excitability of developing DGCs at $21 \mathrm{dpi} . A$, Left panels, Whole-cell recordings of inward-rectifying currents evoked by voltage steps $(5 \mathrm{mV}, 100 \mathrm{~ms})$ from -40 to $-130 \mathrm{mV}$ in DGCs expressing GFP alone (control),

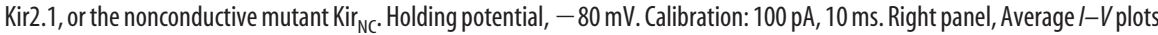
ta shown in $A .{ }^{*} p<0.05$ and ${ }^{* *} p<0.01$ analyzed with Kruskal-Wallis statistics followed by Dunn's multiple-comparison test. (Kir), and 17 neurons (Kir ${ }_{N C}$ ). ${ }^{* *} p<0.00$

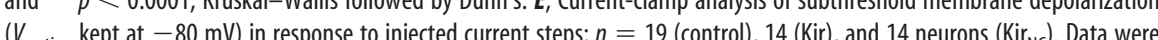
transformed to reach normality using a natural log function. ${ }^{* *} p<0.01$ by two-way ANOVA with Bonferroni's post hoc test. $\boldsymbol{F}$, Suprathreshold responses displaying the number of spikes elicited by depolarizing current steps ( $5 \mathrm{pA}, 400 \mathrm{~ms})$, with $n=14$ (control), 12 (Kir), and 14 neurons (Kir ${ }_{N C}$ ). All data represent mean \pm SEM obtained from three to four mice in each condition.

pocampal regions, such as dendritic trees spanning the entire molecular layer, a round soma, and absence of basal dendrites. At higher magnification, we observed thicker dendritic processes coated with spines in both regions of the dentate gyrus, and the conspicuous varicosities observed in the temporal area of sedentary mice were no longer present in running mice (Fig. 5E). Accordingly, a significant increase in spine density was induced by running exclusively in new DGCs of the temporal region (Fig. $5 F$ ). In agreement with these results, the regional differences in $\mathrm{Cb}$ expression at $21 \mathrm{dpi}$ were also equalized by running. The proportion of newborn DGCs labeled with $\mathrm{Cb}$ was not modified by running in the septal area, but it displayed a threefold increase (to $\sim 75 \%$ ) in the temporal region (Fig. $5 G, H$ ). These observations demonstrate that voluntary exercise can accelerate the rate of neuronal development specifically in the temporal dentate 
A

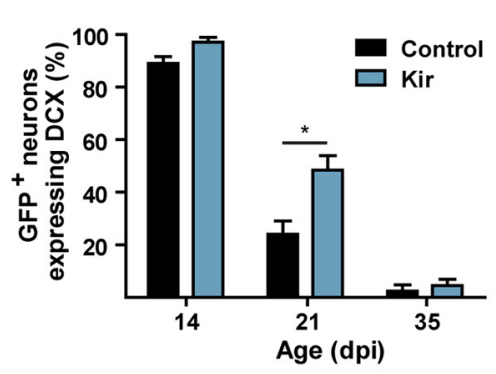

C

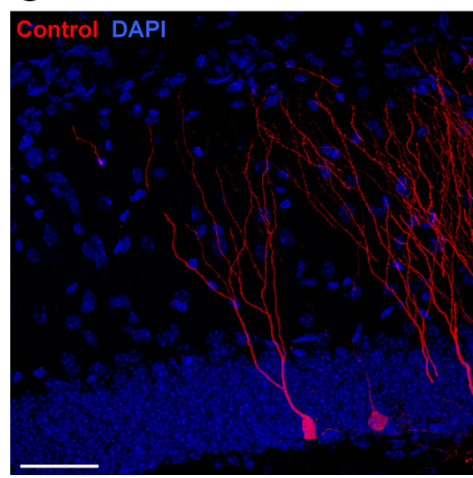

B

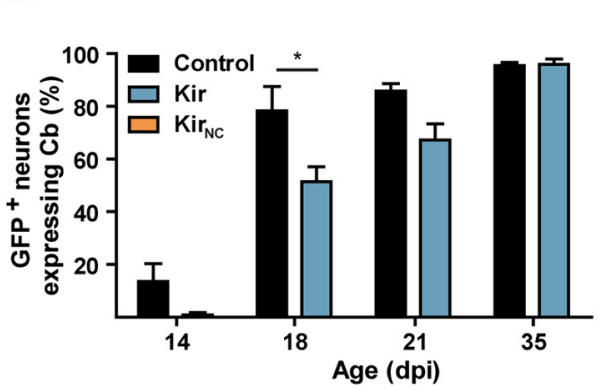

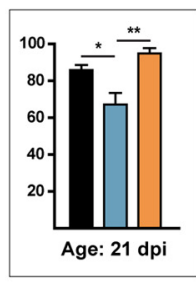

D

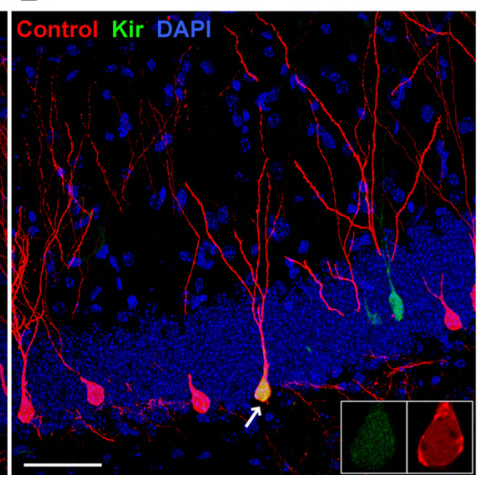

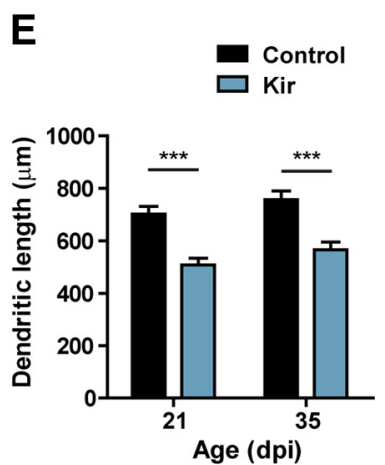

Figure 7. Reduction of intrinsic excitability delays maturation of newborn granule cells in running mice. $\boldsymbol{A}$, Prolonged DCX expression in newborn DGCs expressing Kir. Each bar represents data obtained counting a total of 120-350 neurons from $n=3-5$ mice. ${ }^{*} p<0.05$. B, Delayed onset of Cb expression in adult-born DGCs expressing Kir. ${ }^{*} p<0.05$. Each bar represents data obtained counting a total of $64-250$ neurons from $n=3-5$ mice. Inset, Cb expression at 21 dpi including the comparison with new DGCs expressing the mutant nonconductive Kir ${ }_{\mathrm{NC} .}{ }^{* *} p<0.01$. $\boldsymbol{C}, \boldsymbol{D}$, Three-week-old neurons expressing the fluorescent reporter alone (RFP, red) or coinfected with Kir tagged with GFP (green). DAPI (blue) was used to label the GCL. Scale bars, $50 \mu \mathrm{m}$. Inset, Single confocal planes of the separate red and green channels for the coinfected neuron pointed by the arrow. $\boldsymbol{E}$, Quantitative analysis of the total dendritic length of newborn DGCs at the indicated times. ${ }^{* * *} p<0.001$ with control, $n=14-20$ neurons obtained from five to eight mice. Bars denote mean \pm SEM. All statistical comparisons were assessed by two-way ANOVA followed by Bonferroni's post hoc test.

gyrus. In these conditions, maturation of newborn DGCs in the septal and temporal hippocampus occurs at similar rates.

\section{A critical role for intrinsic activity on neuronal maturation}

The experiments presented above highlight a correlation between the level of activity in the neurogenic niche and the rate of neuronal development and functional integration. We next evaluated whether there is a causal link between electrical activity and neuronal maturation. To test whether neuronal activity can directly modulate neuronal maturation, excitability of newborn DGCs was decreased by retroviral delivery of the inward rectifier $\mathrm{K}^{+}$channel Kir2.1. Retrovirally labeled neurons expressing GFP (control) or a mutant Kir2.1 with a nonconductive pore $\left(\operatorname{Kir}_{\mathrm{NC}}\right)$ were used as controls. Electrophysiological recordings confirmed that 21 dpi neurons overexpressing Kir (but not $\operatorname{Kir}_{\mathrm{NC}}$ ) displayed larger inward rectifier currents (approximately threefold increase), decreased input resistance, hyperpolarized resting potential, and diminished levels of subthreshold depolarization (Fig. 6). These results demonstrate that Kir overexpression can efficiently reduce the excitability of developing DGCs.

Phenotypic effects of a decrease in the intrinsic activity of developing neurons were analyzed at the morphological and physiological level. These experiments were performed in running mice to equalize basal activity at the maximal level for both the septal and temporal regions. New neurons overexpressing Kir almost doubled DCX expression at $21 \mathrm{dpi}$ and displayed reduced $\mathrm{Cb}$ levels between 14 and 21 dpi when compared with control DGCs (Fig. $7 A, B$ ). Notably, Cb immunoreactivity in 21 dpi neurons expressing Kir $_{\mathrm{NC}}$ was similar to control DGCs (Fig. 7B, inset), indicating that the phenotypic defect induced by Kir is specific to the reduced excitability. At 35 dpi, DCX was absent and $\mathrm{Cb}$ expression was maximal in Kir and control new neurons, indicating that Kir neurons have reached a mature pattern of marker expression. These results demonstrate that reduced intrinsic excitability delayed the rate of newborn cell maturation in a highly active environment.

In addition to the delayed expression of early and late markers, Kir neurons displayed short dendrites at $21 \mathrm{dpi}$, also consistent with a more immature neuronal stage (Fig. 7C-E). This morphological difference was still present at $35 \mathrm{dpi}$, highlighting an additional delay that is maintained beyond the timing required for $\mathrm{Cb}$ expression. To determine whether the diminished dendritic length is reflected at the level of neuronal connectivity, we performed recordings of mEPSCs and mIPSCs. New DGCs expressing Kir displayed lower frequency of both mEPSCs and mIPSCs than control cells with little or no differences in amplitude, reflecting poor afferent connectivity (Fig. $8 A-C$ ). Finally, new neurons expressing Kir (but not $\mathrm{Kir}_{\mathrm{NC}}$ ) evidenced a striking reduction in cell membrane capacitance $\left(C_{\mathrm{m}}\right)$, a parameter that is proportional to the area of the neuronal membrane (Fig. $8 D$ ). The smaller $C_{\mathrm{m}}$ in Kir neurons is also consistent with a more immature stage of development (Espósito et al., 2005). These observations indicate that intrinsic excitability regulates the maturation of newborn DGCs in the adult hippocampus. We conclude that electrical activity in the neurogenic niche plays a crucial role in the timing required for neuronal maturation.

\section{Discussion}

Developing DGCs of the adult hippocampus undergo well defined transitions in morphology, excitability, and connectivity in 
the pathway toward a fully mature phenotype (Ambrogini et al., 2004; Espósito et al., 2005; Overstreet-Wadiche et al., 2005, 2006a; Ge et al., 2006; Laplagne et al., 2006; Piatti et al., 2006; Zhao et al., 2006; Toni et al., 2007, 2008). Among those transitions, there is a window for the expression of unique properties that establishes newborn DGCs as a distinctive functional population within the adult dentate gyrus (Wang et al., 2000; Snyder et al., 2001; Schmidt-Hieber et al., 2004; Ge et al., 2007; Mongiat et al., 2009). In addition, the view that the hippocampus contains heterogeneous niches for adult neurogenesis is now clearly emerging. Recent studies have shown that larger pools of newborn DGCs are generated in the septal region of the adult dentate gyrus compared with the temporal pole (Tashiro et al., 2007; Snyder et al., 2009a,b; Jinno, 2011). Such septotemporal differences in neurogenesis are most likely attributable to higher rates of progenitor cell proliferation or neuronal survival in the more active septal area. In this work, we demonstrate that the hippocampus also contains heterogeneous domains in regard to the rate of neuronal maturation, with the septal and temporal areas exhibiting different features. Our experiments in sedentary mice reveal that newborn DGCs are ready to participate in information processing in the septal hippocampus at a time when same-cohort neurons of the temporal region are still highly immature and poorly connected. Moreover, the septotemporal difference in the timing of neuronal maturation described in this study predicts a shorter period of enhanced excitability in the septal dentate gyrus. This functional polarization of the hippocampus is tightly associated to differences in the basal level of activity in the local environment. We also show that running increases local activity and, consequently, accelerates neuronal maturation only in the temporal dentate gyrus to a point in which septotemporal differences are equalized. We finally show that intrinsic neuronal activity of developing DGCs is crucial for shaping the timing for maturation.

\section{Neuronal activity modulates the timing of maturation of newborn DGCs}

Voluntary locomotion was known to be coupled to neuronal activity in the hippocampus (Whishaw and Vanderwolf, 1973). In fact, the discharge rate of hippocampal pyramidal cells and interneurons was shown to increase with the running velocity even under conditions in which the animal was held in a fixed space but allowed to run (Czurkó et al., 1999; Maurer et al., 2005). In agreement, we observed that running increased the number of active neurons in the GCL. However, the fact that an increased proportion of active DGCs was only found in the temporal but

D
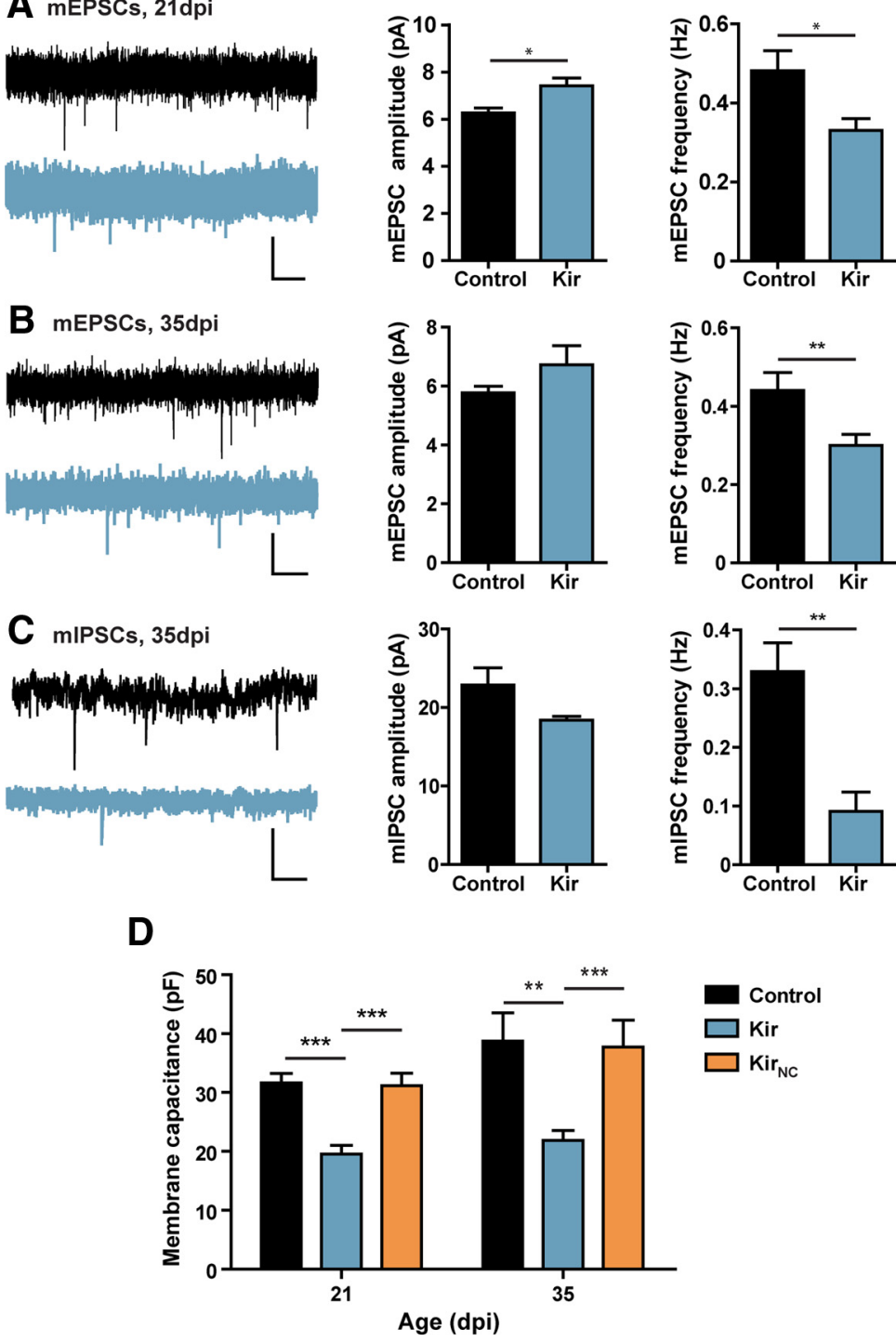

Figure 8. Kir2.1 overexpression impairs functional connectivity of newborn DGCs in running mice. $A$, Analysis of mEPSCs for 21 dpi neurons. Left panels show example voltage-clamp whole-cell recordings obtained from a GFP-expressing neuron (control, black) and a Kir-overexpressing neuron (blue). Right panels depict quantitative analysis of mEPSC amplitude and frequency; $n=$ of mEPSCs for 35 dpi neurons, with $n=14$ for both control and Kir neurons. ${ }^{* *} p=0.0094$, Mann-Whitney test. Calibration: $5 \mathrm{pA}$ , Analysis of mIPSCs for 35 dpi neurons, with $n=12$ (control) and $n=8$ (Kir). ${ }^{* *} p=0.0018$, Mann-Whitney test. Calibration:

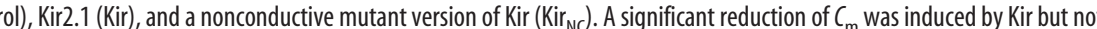
to time points. Data for 35 dpi were transformed to reach normality using a natural $\log$ function. ${ }^{* *} p<0.01$ and ${ }^{* * *} p<$ 0.001 by ANOVA with Bonferroni's posthoc test, with $n=41$ ( $21 \mathrm{dpi}$, control), 41 (21 dpi, Kir), 17 (21 dpi, Kir ${ }_{\mathrm{NC}}$ ), 15 (35 dpi, control), 15 ( $35 \mathrm{dpi}$, Kir), and 11 (35 dpi, Kir ${ }_{\mathrm{NC}}$. All bar charts represent mean \pm SEM.

not the septal dentate gyrus is surprising. Regional differences in the inhibitory network might contribute to the differential responsiveness to running. The number of inhibitory basket cells per DGCs is higher in the septal than the temporal dentate gyrus (Seress and Pokorny, 1981). In addition, $\mathrm{CA}_{1}$ pyramidal cells of both regions increased their firing rate as a function of running speed, whereas the spiking rate of $\mathrm{CA}_{1}$ interneurons was always higher in the septal region (Maurer et al., 2005).

However, previous studies have reported different levels of activity measured by Arc expression across the infrapyramidal and suprapyramidal blades of the rat granule cell layer after exploration or learning but not in caged controls (Chawla et al., 2005; Ramirez-Amaya et al., 2006; Snyder et al., 2009a). In agree- 


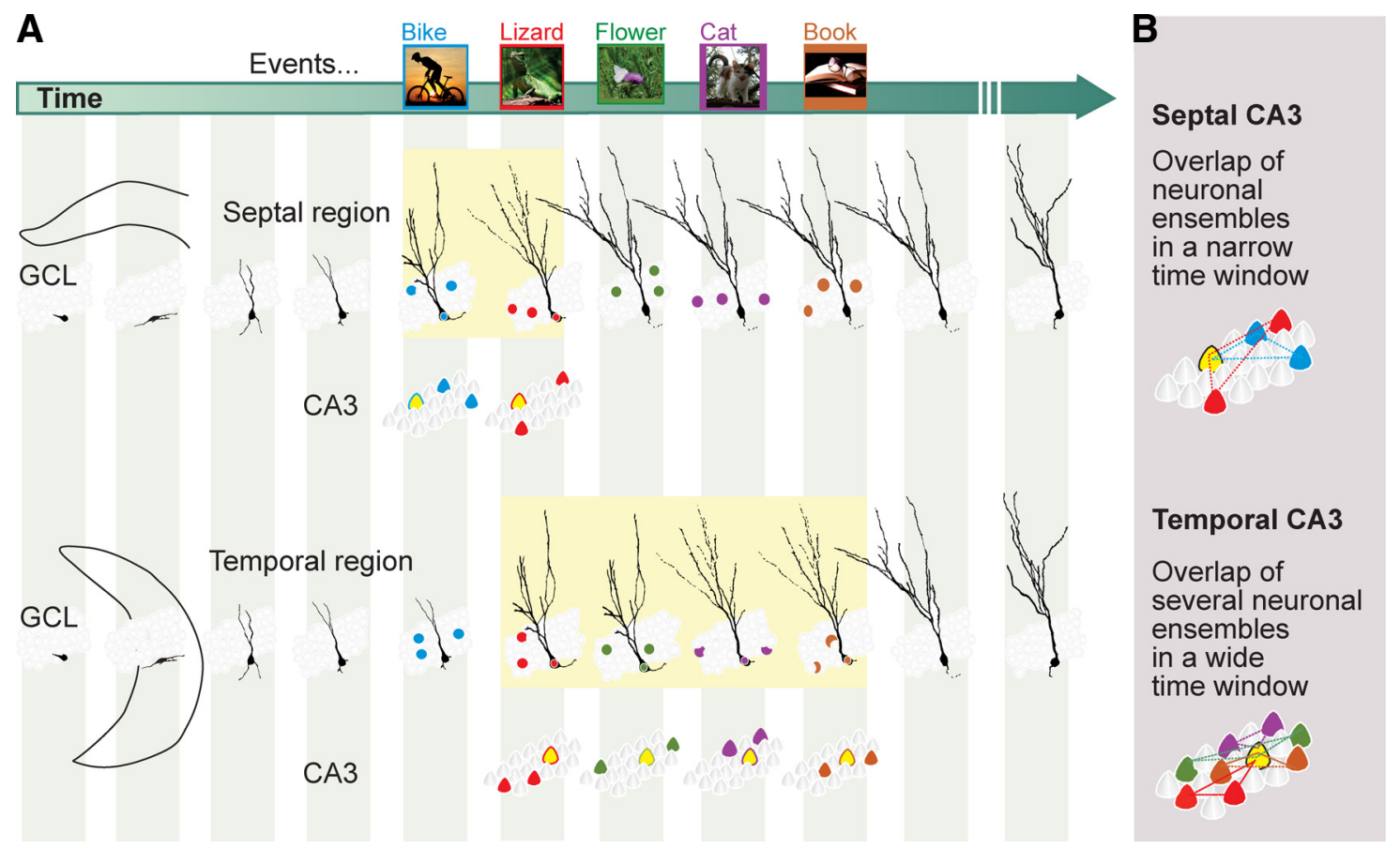

Figure 9. Potential role for the differential timing of neuronal maturation in memory encoding. $A$, Time sequence ( $g$ ray columns) of neuronal maturation represented by two adult-born neurons from the same cohort developing at different rates in the septal and temporal dentate gyrus. The period of enhanced excitability for each developing neuron is represented by yellow boxes, with recruited $\mathrm{CA}_{3}$ ensembles shown below. As a result of the faster maturation rate, the period of enhanced excitability is shorter in the septal region than in the temporal area. Therefore, for events happening close in time, such as biking one day (blue) and finding a lizard the following day (red), the same immature neuron of the septal region would be active, together with the mature neuronal ensembles representing these events (colored neurons). In contrast, the immature neuron developing in the temporal dentate gyrus would be active together with neuronal ensembles representing events occurring within a longer interval, such as finding a lizard (red) that day, seeing a butterfly on a flower (green), looking at a cat on a roof (purple), and finishing a book (brown) during consecutive days. Note that the fast-maturing neuron in the septal region is active during two events, whereas the slow-maturing neuron in the temporal area is active for four ensembles. As a consequence, in the downstream $\mathrm{CA}_{3}$ region, the same pyramidal neuron (yellow, target of new neurons) will be recruited to be part of two ensembles (blue and red) in the septal region but four ensembles (red, green, purple, and brown) in the temporal area. $B, C_{3}$ ensembles (memory representations) for different events may show varying amounts of overlap depending on whether upstream DGCs developed in the septal or temporal regions: septal $\mathrm{CA}_{3}$ cells would show less overlap (the yellow cell is part of two ensembles), whereas temporal $C \mathrm{~A}_{3}$ cells would show more overlap (the yellow cell is part of four ensembles). In this example, activation of the blue ensemble in the septal region might activate the red ensemble, such that the memory of "finding a lizard" would be retrieved during the "biking" recall. In contrast, activation of the red ensemble in the temporal region could activate several neuronal ensembles that might provide contextual information such as the time of "reading a book."

ment, we found no differences in the activity of the suprapyramidal vs infrapyramidal blades neither for the septal nor the temporal dentate gyrus in sedentary or running mice. Sedentary mice displayed significant differences in Arc expression for septal versus temporal areas $(p<0.0001)$ but not for suprapyramidal versus infrapyramidal blades ( $p=0.19$, two-way ANOVA; $n=9$ mice). Interestingly, septotemporal differences on neuronal activity were equalized in running mice without differential effects on suprapyramidal versus infrapyramidal blades $(p>0.5$ for both, two-way ANOVA; $N=9$ mice). Therefore, both blades of the temporal dentate gyrus respond to voluntary movement in chronically running mice, in agreement with the higher involvement of the temporal hippocampus in sensorimotor processes (Bast and Feldon, 2003; Bast et al., 2009).

Interestingly, the rate of neuronal maturation correlated with the pattern of neuronal activity. Low levels of basal activity such as those seen in the temporal dentate gyrus of sedentary mice support a slow pace of development for newborn cells. In contrast, high network activity such as that found in the septal dentate gyrus of sedentary mice and in both septal and temporal areas of running mice promotes a higher rate of maturation. Finally, we found no differences in sedentary or running mice in the degree of maturation of neurons developing in different blades of the same dentate gyrus region as assessed by calbindin and DCX expression at $21 \mathrm{dpi}$ (data not shown), supporting our conclusion that local network activity modulates neuronal development.
To investigate whether neuronal activity can directly modulate maturation, we analyzed the effect of decreased excitability by retroviral delivery of Kir2.1 into developing DGCs in running mice, the most active niche in our experimental conditions, in which basal activity and neuronal maturation in the septal and temporal dentate gyrus are equalized. Kir overexpression induced a significant reduction in the excitability of developing DGCs, yet neurons were not completely silenced. This moderate reduction in excitability induced a remarkable delay in maturation. Neurons expressing Kir exhibited prolonged expression of DCX and delayed onset of $\mathrm{Cb}$ when compared with control DGCs, and the dendritic tree and afferent connectivity remained diminished even after $35 \mathrm{~d}$. It is noteworthy that newborn neurons mature over several weeks, yet little change was observed in mEPSC frequency and dendritic length in control DGCs between 21 and 35 dpi (Figs. 7E, $8 A, B$ ). This observation is consistent with our previous findings that the strength of excitatory connectivity increases abruptly at $\sim 3$ and $\sim 6$ weeks but remains unchanged within this time window (Mongiat et al., 2009). We speculate that neuronal connectivity might undergo extensive synaptogenesis and pruning during this interval (Shen and Scheiffele, 2010). Together, our results demonstrate that DGCs with reduced levels of intrinsic activity mature slower than those that are more active. This notion is consistent with the slower rate of maturation observed in the temporal dentate gyrus of sedentary mice. We conclude that the overall level of activity of the network determines 
the level of activity of each developing neuron and, consequently, controls the time during which newborn DGCs remain immature. We therefore propose activity-dependent modulation of neuronal maturation as a novel type of hippocampal plasticity.

Recent evidence has implicated electrical activity in the survival of adult-born neurons both in the hippocampus and olfactory bulb $(\mathrm{OB})$. In the hippocampus, NMDA receptor activation promotes integration and survival of newborn DGCs (BruelJungerman et al., 2006; Tashiro et al., 2006). In the OB, NMDAmediated signaling is critical for neuroblast survival during migration along the rostral migratory stream (Platel et al., 2010). After migration has ended, intrinsic activity promotes the survival of neurons that are integrating in the OB circuitry (Lin et al., 2010). In addition, it was shown that altering the level of intrinsic activity did not affect the development of input or output connectivity in newborn cells of the OB (Kelsch et al., 2009). In contrast, our findings demonstrate that electrical activity greatly influences the rate of neuronal maturation and functional integration in the hippocampus.

\section{Possible mechanisms underlying activity-dependent changes in neuronal maturation}

In principle, the septotemporal differences in neuronal maturation might be determined in a cell-autonomous fashion, similarly to the distinct neuronal phenotypes arising from neural progenitor cells along the adult subventricular zone (Merkle et al., 2007). However, our observations highlight niche differences generated by the level of network activity as a primary modulatory mechanism that determines the rate of neuronal maturation. Our results demonstrate that the septal and temporal dentate gyrus display distinct activity set points that impinge on the rate of neuronal development. Changing those set points by running produced striking effects on maturation. Remarkably, those activity-dependent modifications occurred under physiological conditions.

Local network activity could modulate neuronal maturation by acting directly within developing neurons or by inducing secretion of factors from mature neighboring cells (Zweifel et al., 2005; Spitzer, 2006; Flavell and Greenberg, 2008). For instance, synthesis and secretion of neurotrophic factors such as brainderived neurotrophic factor (BDNF), which participates in neuronal differentiation during adult neurogenesis, can be locally increased by electrical activity (Cotman and Berchtold, 2002; Bergami et al., 2008; Ma et al., 2009). In this work, we demonstrate that decreasing neuronal excitability by Kir overexpression reduces the rate of neuronal maturation, highlighting the crucial role of intrinsic activity in controlling this process. It is very likely that the effects of activity on maturation are triggered by subthreshold depolarization rather than by firing of action potentials. Consistent with this notion, we have previously shown that newborn DGCs begin to receive afferent excitation after $\sim 15 \mathrm{dpi}$, but they can only generate action potentials in response to an excitatory drive after 24-28 dpi (Mongiat et al., 2009). Intracellular effects of depolarizing activity might be mediated by the CREB signaling pathway, which has recently been involved in dendritic development and survival of adult-born DGCs (Jagasia et al., 2009). Therefore, BDNF and other released factors might act synergistically with intrinsic activity to shape neuronal maturation in the adult hippocampus, similarly to what has been described for BDNF actions on synaptic plasticity (Schinder and Poo, 2000).

\section{Relevance of activity-dependent neuronal maturation in hippocampal function}

The hippocampus is critical for episodic memory, forming unique memory traces about what, where, and when an event has occurred (Scoville and Milner, 1957; Ergorul and Eichenbaum, 2004). The dentate gyrus is the first processing stage of the hippocampus and it plays a critical role on the discrimination of similar visuospatial inputs, a necessary step for encoding episodic memory (Leutgeb et al., 2007). Adult neurogenesis in the dentate gyrus has been conserved through vertebrate evolution, and it may offer functional advantages because of the unique properties of young neurons (Treves et al., 2008). It was recently shown that developing DGCs display enhanced excitability and synaptic plasticity (Wang et al., 2000; Schmidt-Hieber et al., 2004; Ge et al., 2007; Mongiat et al., 2009). In addition, a relevant hypothesis on episodic memory formation has proposed that immature neurons would be activated by a broad range of stimuli because of their increased excitability, allowing association of temporally related events (Aimone et al., 2006). We propose two novel features to the current model that could modulate episodic memory encoding according to the animal's experience. First, the differential rate of neuronal maturation could provide different scales for encoding temporally related events in the septal and temporal dentate gyrus (Fig. 9). The shorter intervals of neuronal maturation in the septal region could be involved in highly detailed representations that demand a compressed timescale, whereas the prolonged window of maturation in the temporal region would provide an expanded scale for time associations of incoming inputs involving more generalized representations. Finally, network activity could shape these timescales of neuronal representation by modulating the timing for the expression of those unique functional properties acting at the level of neuronal maturation. Accelerating neuronal maturation could enhance temporal resolution and reliability on the encoding of multiple events by a high number of new neurons that are fast developing in the active network.

\section{References}

Abrous DN, Koehl M, Le Moal M (2005) Adult neurogenesis: from precursors to network and physiology. Physiol Rev 85:523-569.

Aimone JB, Wiles J, Gage FH (2006) Potential role for adult neurogenesis in the encoding of time in new memories. Nat Neurosci 9:723-727.

Amaral DG, Witter MP (1989) The three-dimensional organization of the hippocampal formation: a review of anatomical data. Neuroscience 31:571-591.

Ambrogini P, Lattanzi D, Ciuffoli S, Agostini D, Bertini L, Stocchi V, Santi S, Cuppini R (2004) Morpho-functional characterization of neuronal cells at different stages of maturation in granule cell layer of adult rat dentate gyrus. Brain Res 1017:21-31.

Bannerman DM, Rawlins JN, McHugh SB, Deacon RM, Yee BK, Bast T, Zhang WN, Pothuizen HH, Feldon J (2004) Regional dissociations within the hippocampus - memory and anxiety. Neurosci Biobehav Rev 28:273-283.

Bast T, Feldon J (2003) Hippocampal modulation of sensorimotor processes. Prog Neurobiol 70:319-345.

Bast T, Wilson IA, Witter MP, Morris RG (2009) From rapid place learning to behavioral performance: a key role for the intermediate hippocampus. PLoS Biol 7:e1000089.

Bayer SA (1982) Changes in the total number of dentate granule cells in juvenile and adult rats: a correlated volumetric and ${ }^{3} \mathrm{H}$-thymidine autoradiographic study. Exp Brain Res 46:315-323.

Bergami M, Rimondini R, Santi S, Blum R, Götz M, Canossa M (2008) Deletion of TrkB in adult progenitors alters newborn neuron integration into hippocampal circuits and increases anxiety-like behavior. Proc Natl Acad Sci U S A 105:15570-15575.

Bruel-Jungerman E, Davis S, Rampon C, Laroche S (2006) Long-term po- 
tentiation enhances neurogenesis in the adult dentate gyrus. J Neurosci 26:5888-5893.

Burrone J, O’Byrne M, Murthy VN (2002) Multiple forms of synaptic plasticity triggered by selective suppression of activity in individual neurons. Nature 420:414-418.

Chawla MK, Guzowski JF, Ramirez-Amaya V, Lipa P, Hoffman KL, Marriott LK, Worley PF, McNaughton BL, Barnes CA (2005) Sparse, environmentally selective expression of Arc RNA in the upper blade of the rodent fascia dentata by brief spatial experience. Hippocampus 15:579-586.

Cotman CW, Berchtold NC (2002) Exercise: a behavioral intervention to enhance brain health and plasticity. Trends Neurosci 25:295-301.

Czurkó A, Hirase H, Csicsvari J, Buzsáki G (1999) Sustained activation of hippocampal pyramidal cells by "space clamping" in a running wheel. Eur J Neurosci 11:344-352.

Dayer AG, Ford AA, Cleaver KM, Yassaee M, Cameron HA (2003) Shortterm and long-term survival of new neurons in the rat dentate gyrus. J Comp Neurol 460:563-572.

Dolorfo CL, Amaral DG (1998) Entorhinal cortex of the rat: organization of intrinsic connections. J Comp Neurol 398:49-82.

Ergorul C, Eichenbaum H (2004) The hippocampus and memory for "what," "where," and "when." Learn Mem 11:397-405.

Espósito MS, Piatti VC, Laplagne DA, Morgenstern NA, Ferrari CC, Pitossi FJ, Schinder AF (2005) Neuronal differentiation in the adult hippocampus recapitulates embryonic development. J Neurosci 25:10074-10086.

Fanselow MS, Dong HW (2010) Are the dorsal and ventral hippocampus functionally distinct structures? Neuron 65:7-19.

Flavell SW, Greenberg ME (2008) Signaling mechanisms linking neuronal activity to gene expression and plasticity of the nervous system. Annu Rev Neurosci 31:563-590.

Foster TC, Castro CA, McNaughton BL (1989) Spatial selectivity of rat hippocampal neurons: dependence on preparedness for movement. Science 244:1580-1582.

Fyhn M, Molden S, Witter MP, Moser EI, Moser MB (2004) Spatial representation in the entorhinal cortex. Science 305:1258-1264.

Ge S, Goh EL, Sailor KA, Kitabatake Y, Ming GL, Song H (2006) GABA regulates synaptic integration of newly generated neurons in the adult brain. Nature 439:589-593.

Ge S, Yang CH, Hsu KS, Ming GL, Song H (2007) A critical period for enhanced synaptic plasticity in newly generated neurons of the adult brain. Neuron 54:559-566.

Gould E, Beylin A, Tanapat P, Reeves A, Shors TJ (1999) Learning enhances adult neurogenesis in the hippocampal formation. Nat Neurosci 2:260-265.

Jagasia R, Steib K, Englberger E, Herold S, Faus-Kessler T, Saxe M, Gage FH, Song H, Lie DC (2009) GABA-cAMP response element-binding protein signaling regulates maturation and survival of newly generated neurons in the adult hippocampus. J Neurosci 29:7966-7977.

Jinno S (2011) Topographic differences in adult neurogenesis in the mouse hippocampus: a stereology-based study using endogenous markers. Hippocampus 21:467-480.

Jones SP, Rahimi O, O’Boyle MP, Diaz DL, Claiborne BJ (2003) Maturation of granule cell dendrites after mossy fiber arrival in hippocampal field CA3. Hippocampus 13:413-427.

Jung MW, Wiener SI, McNaughton BL (1994) Comparison of spatial firing characteristics of units in dorsal and ventral hippocampus of the rat. J Neurosci 14:7347-7356.

Kee N, Teixeira CM, Wang AH, Frankland PW (2007) Preferential incorporation of adult-generated granule cells into spatial memory networks in the dentate gyrus. Nat Neurosci 10:355-362.

Kelsch W, Lin CW, Mosley CP, Lois C (2009) A critical period for activitydependent synaptic development during olfactory bulb adult neurogenesis. J Neurosci 29:11852-11858.

Kjelstrup KG, Tuvnes FA, Steffenach HA, Murison R, Moser EI, Moser MB (2002) Reduced fear expression after lesions of the ventral hippocampus. Proc Natl Acad Sci U S A 99:10825-10830.

Laplagne DA, Espósito MS, Piatti VC, Morgenstern NA, Zhao C, van Praag H, Gage FH, Schinder AF (2006) Functional convergence of neurons generated in the developing and adult hippocampus. PLoS Biol 4:e409.

Laplagne DA, Kamienkowski JE, Espósito MS, Piatti VC, Zhao C, Gage FH, Schinder AF (2007) Similar GABAergic inputs in dentate granule cells born during embryonic and adult neurogenesis. Eur J Neurosci 25:2973-2981.
Leutgeb JK, Leutgeb S, Moser MB, Moser EI (2007) Pattern separation in the dentate gyrus and CA3 of the hippocampus. Science 315:961-966.

Lin CW, Sim S, Ainsworth A, Okada M, Kelsch W, Lois C (2010) Genetically increased cell-intrinsic excitability enhances neuronal integration into adult brain circuits. Neuron 65:32-39.

Ma DK, Jang MH, Guo JU, Kitabatake Y, Chang ML, Pow-Anpongkul N, Flavell RA, Lu B, Ming GL, Song H (2009) Neuronal activity-induced Gadd45b promotes epigenetic DNA demethylation and adult neurogenesis. Science 323:1074-1077.

Markwardt SJ, Wadiche JI, Overstreet-Wadiche LS (2009) Input-specific GABAergic signaling to newborn neurons in adult dentate gyrus. J Neurosci 29:15063-15072.

Maurer AP, Vanrhoads SR, Sutherland GR, Lipa P, McNaughton BL (2005) Self-motion and the origin of differential spatial scaling along the septotemporal axis of the hippocampus. Hippocampus 15:841-852.

Merkle FT, Mirzadeh Z, Alvarez-Buylla A (2007) Mosaic organization of neural stem cells in the adult brain. Science 317:381-384.

Mongiat LA, Espósito MS, Lombardi G, Schinder AF (2009) Reliable activation of immature neurons in the adult hippocampus. PLoS One 4:e5320.

Morgenstern NA, Lombardi G, Schinder AF (2008) Newborn granule cells in the aging dentate gyrus. J Physiol 586:3751-3757.

Moser MB, Moser EI, Forrest E, Andersen P, Morris RG (1995) Spatial learning with a minislab in the dorsal hippocampus. Proc Natl Acad Sci U S A 92:9697-9701.

Overstreet-Wadiche L, Bromberg DA, Bensen AL, Westbrook GL (2005) GABAergic signaling to newborn neurons in dentate gyrus. J Neurophysiol 94:4528-4532.

Overstreet-Wadiche LS, Bensen AL, Westbrook GL (2006a) Delayed development of adult-generated granule cells in dentate gyrus. J Neurosci 26:2326-2334.

Overstreet-Wadiche LS, Bromberg DA, Bensen AL, Westbrook GL (2006b) Seizures accelerate functional integration of adult-generated granule cells. J Neurosci 26:4095-4103.

Parent JM, Lowenstein DH (2002) Seizure-induced neurogenesis: are more new neurons good for an adult brain? Prog Brain Res 135:121-131.

Paxinos G, Franklin KBJ (1997) The mouse brain in stereotaxic coordinates. San Diego: Academic.

Petrovich GD, Canteras NS, Swanson LW (2001) Combinatorial amygdalar inputs to hippocampal domains and hypothalamic behavior systems. Brain Res Brain Res Rev 38:247-289.

Piatti VC, Espósito MS, Schinder AF (2006) The timing of neuronal development in adult hippocampal neurogenesis. Neuroscientist 12:463-468.

Platel JC, Dave KA, Gordon V, Lacar B, Rubio ME, Bordey A (2010) NMDA receptors activated by subventricular zone astrocytic glutamate are critical for neuroblast survival prior to entering a synaptic network. Neuron 65:859-872.

Ramirez-Amaya V, Marrone DF, Gage FH, Worley PF, Barnes CA (2006) Integration of new neurons into functional neural networks. J Neurosci 26:12237-12241.

Schinder AF, Poo M (2000) The neurotrophin hypothesis for synaptic plasticity. Trends Neurosci 23:639-645.

Schmidt-Hieber C, Jonas P, Bischofberger J (2004) Enhanced synaptic plasticity in newly generated granule cells of the adult hippocampus. Nature 429:184-187.

Scoville WB, Milner B (1957) Loss of recent memory after bilateral hippocampal lesions. J Neurol Neurosurg Psychiatry 20:11-21.

Seress L, Pokorny J (1981) Structure of the granular layer of the rat dentate gyrus. A light microscopic and Golgi study. J Anat 133:181-195.

Shen K, Scheiffele P (2010) Genetics and cell biology of building specific synaptic connectivity. Annu Rev Neurosci 33:473-507.

Snyder JS, Kee N, Wojtowicz JM (2001) Effects of adult neurogenesis on synaptic plasticity in the rat dentate gyrus. J Neurophysiol 85:2423-2431.

Snyder JS, Radik R, Wojtowicz JM, Cameron HA (2009a) Anatomical gradients of adult neurogenesis and activity: young neurons in the ventral dentate gyrus are activated by water maze training. Hippocampus 19:360-370.

Snyder JS, Ramchand P, Rabbett S, Radik R, Wojtowicz JM, Cameron HA (2009b) Septo-temporal gradients of neurogenesis and activity in 13month-old rats. Neurobiol Aging. Advance online publication. Retrieved May 4, 2011. doi:10.1016/j.neurobiolaging.2009.05.022. 
Spitzer NC (2006) Electrical activity in early neuronal development. Nature 444:707-712.

Tashiro A, Sandler VM, Toni N, Zhao C, Gage FH (2006) NMDA-receptormediated, cell-specific integration of new neurons in adult dentate gyrus. Nature 442:929-933.

Tashiro A, Makino H, Gage FH (2007) Experience-specific functional modification of the dentate gyrus through adult neurogenesis: a critical period during an immature stage. J Neurosci 27:3252-3259.

Thompson CL, Pathak SD, Jeromin A, Ng LL, MacPherson CR, Mortrud MT, Cusick A, Riley ZL, Sunkin SM, Bernard A, Puchalski RB, Gage FH, Jones AR, Bajic VB, Hawrylycz MJ, Lein ES (2008) Genomic anatomy of the hippocampus. Neuron 60:1010-1021.

Toni N, Teng EM, Bushong EA, Aimone JB, Zhao C, Consiglio A, van Praag H, Martone ME, Ellisman MH, Gage FH (2007) Synapse formation on neurons born in the adult hippocampus. Nat Neurosci 10:727-734.

Toni N, Laplagne DA, Zhao C, Lombardi G, Ribak CE, Gage FH, Schinder AF (2008) Neurons born in the adult dentate gyrus form functional synapses with target cells. Nat Neurosci 11:901-907.

Treves A, Tashiro A, Witter ME, Moser EI (2008) What is the mammalian dentate gyrus good for? Neuroscience 154:1155-1172.

Trommald M, Hulleberg G (1997) Dimensions and density of dendritic spines from rat dentate granule cells based on reconstructions from serial electron micrographs. J Comp Neurol 377:15-28.

van Praag H, Kempermann G, Gage FH (1999) Running increases cell proliferation and neurogenesis in the adult mouse dentate gyrus. Nat Neurosci 2:266-270.
Vanderwolf CH (1969) Hippocampal electrical activity and voluntary movement in the rat. Electroencephalogr Clin Neurophysiol 26:407-418.

Vazdarjanova A, Ramirez-Amaya V, Insel N, Plummer TK, Rosi S, Chowdhury S, Mikhael D, Worley PF, Guzowski JF, Barnes CA (2006) Spatial exploration induces ARC, a plasticity-related immediate-early gene, only in calcium/calmodulin-dependent protein kinase II-positive principal excitatory and inhibitory neurons of the rat forebrain. J Comp Neurol 498:317-329.

Verwer RW, Meijer RJ, Van Uum HF, Witter MP (1997) Collateral projections from the rat hippocampal formation to the lateral and medial prefrontal cortex. Hippocampus 7:397-402.

Wang JW, David DJ, Monckton JE, Battaglia F, Hen R (2008) Chronic fluoxetine stimulates maturation and synaptic plasticity of adult-born hippocampal granule cells. J Neurosci 28:1374-1384.

Wang S, Scott BW, Wojtowicz JM (2000) Heterogenous properties of dentate granule neurons in the adult rat. J Neurobiol 42:248-257.

Whishaw IQ, Vanderwolf CH (1973) Hippocampal EEG and behavior: changes in amplitude and frequency of RSA (theta rhythm) associated with spontaneous and learned movement patterns in rats and cats. Behav Biol 8:461-484.

Zhao C, Teng EM, Summers RG Jr, Ming GL, Gage FH (2006) Distinct morphological stages of dentate granule neuron maturation in the adult mouse hippocampus. J Neurosci 26:3-11.

Zhao C, Deng W, Gage FH (2008) Mechanisms and functional implications of adult neurogenesis. Cell 132:645-660.

Zweifel LS, Kuruvilla R, Ginty DD (2005) Functions and mechanisms of retrograde neurotrophin signalling. Nat Rev Neurosci 6:615-625. 